\title{
Exploring the Stability of Mixed-Halide Vacancy-Ordered Quadruple Perovskites
}

\author{
Matthew B. Gray^, Jackson D. Majher^, Noah P. Holzapfel, and Patrick M. Woodward* \\ Department of Chemistry and Biochemistry, The Ohio State University, $100 \mathrm{~W}$. 18th Avenue, \\ Columbus, Ohio 43210, United States
}

\section{$\underline{\text { Supplemental Information }}$}

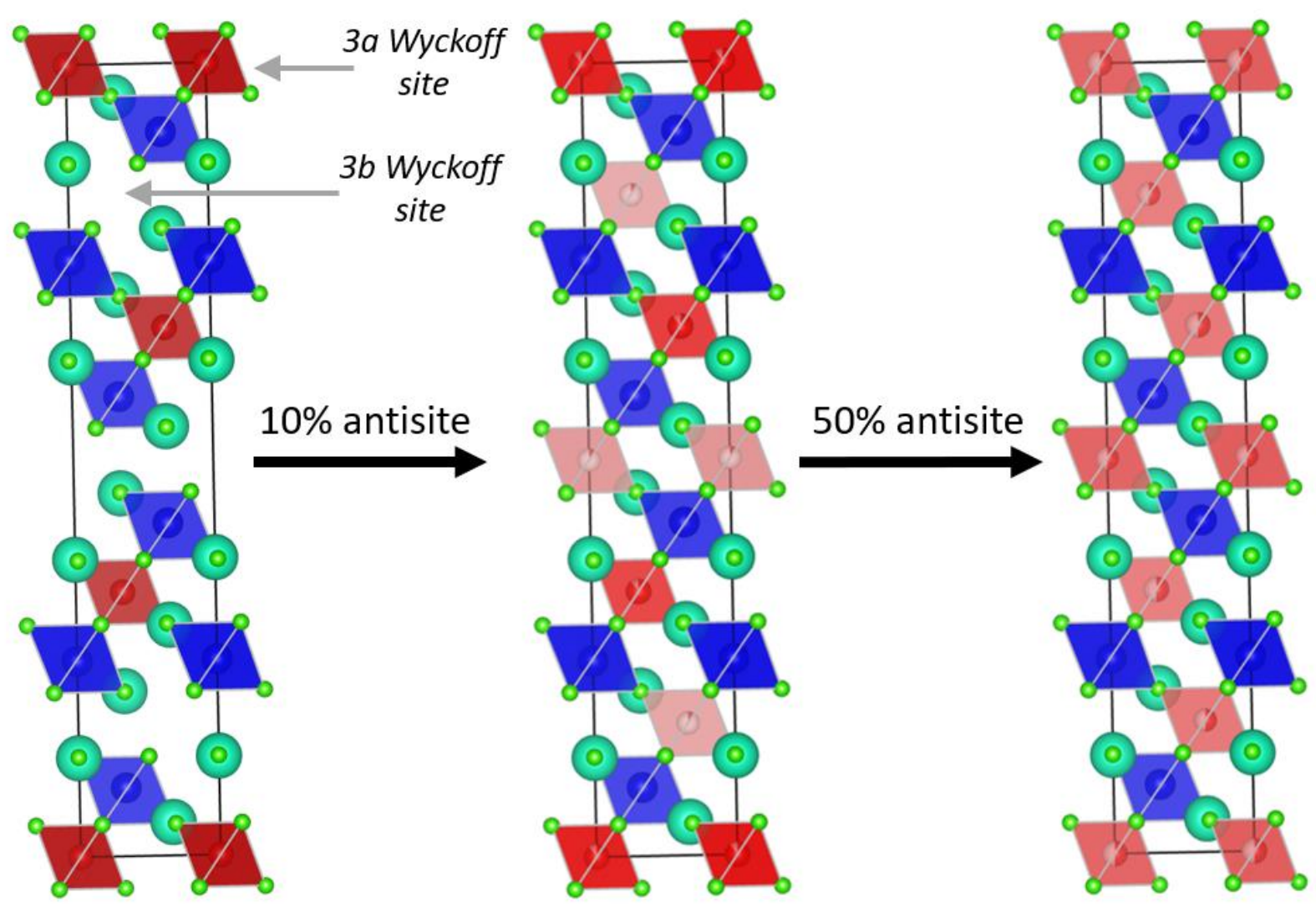

Figure S1. Visualization of the vacancy-ordered quadruple perovskite (VOQP) with 0\% (left), $10 \%$ (middle) and 50\% (right) antisite disorder allowed to refine from PXRD measurements. $\mathrm{M}^{\mathrm{II}}$ and $\mathrm{M}^{\mathrm{III}}$ cations shown as red and blue octahedra, respectively. The depth of red-shading is used to signify the occupation of $\mathrm{M}^{\mathrm{II}}$ ions on either the $3 \mathrm{a}$ or $3 \mathrm{~b}$ Wyckoff site. The maximum antisite disorder observed in this study is $23 \%$ in the $\mathrm{Cs}_{4} \mathrm{CdSb}_{2} \mathrm{Cl}_{12}{ }_{2} \mathrm{Br}_{\mathrm{z}}$ system. 
$\mathrm{Cs}_{4} \mathrm{CdBi}_{2} \mathrm{Cl}_{12-\mathrm{z}} \mathrm{Br}_{\mathrm{z}}$

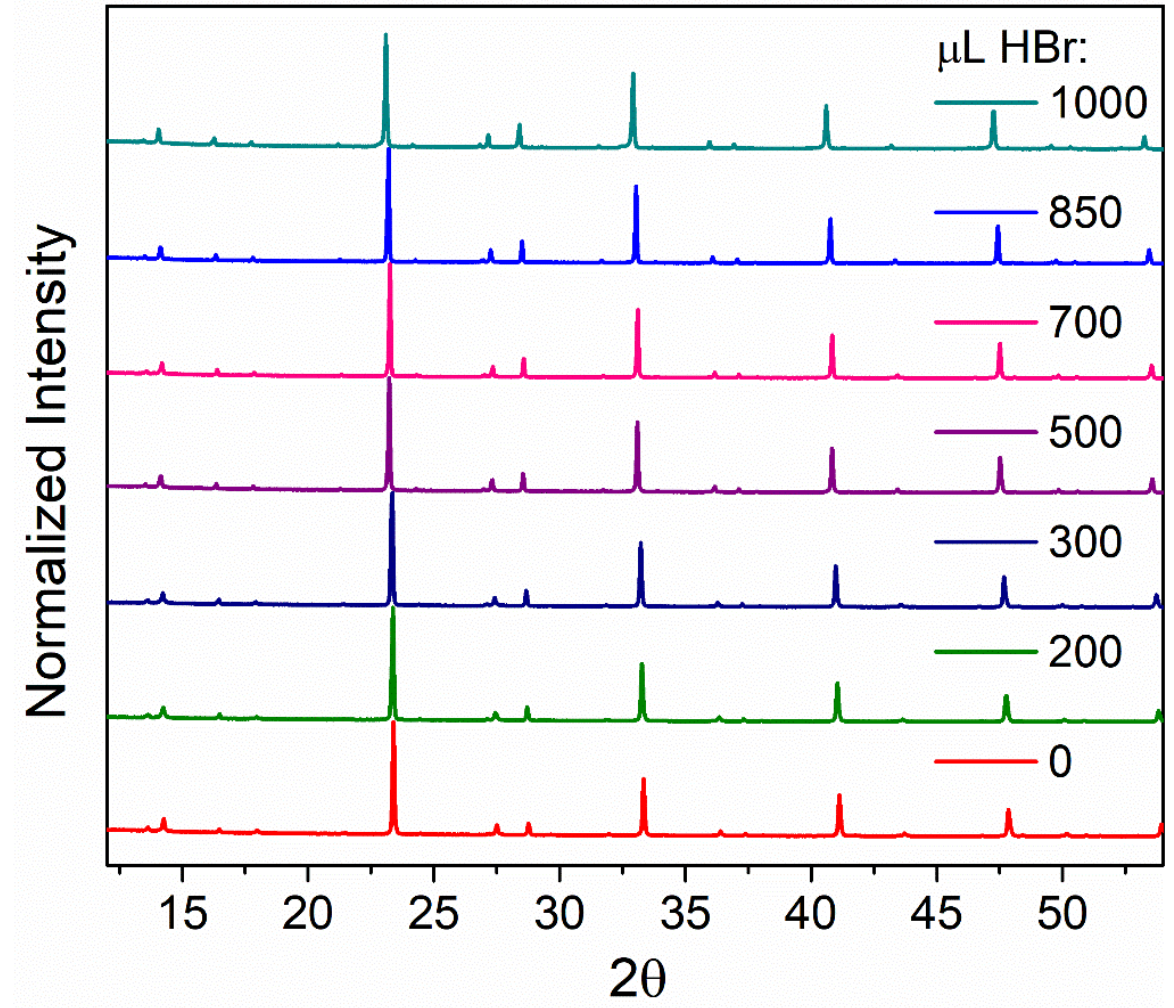

Figure S2. Stacked PXRD patterns of $\mathrm{Cs}_{4} \mathrm{CdBi}_{2} \mathrm{Cl}_{12-} \mathrm{Br}_{2}$ specimens prepared from solutions containing varying amounts of $\operatorname{HBr}(a q)$. 


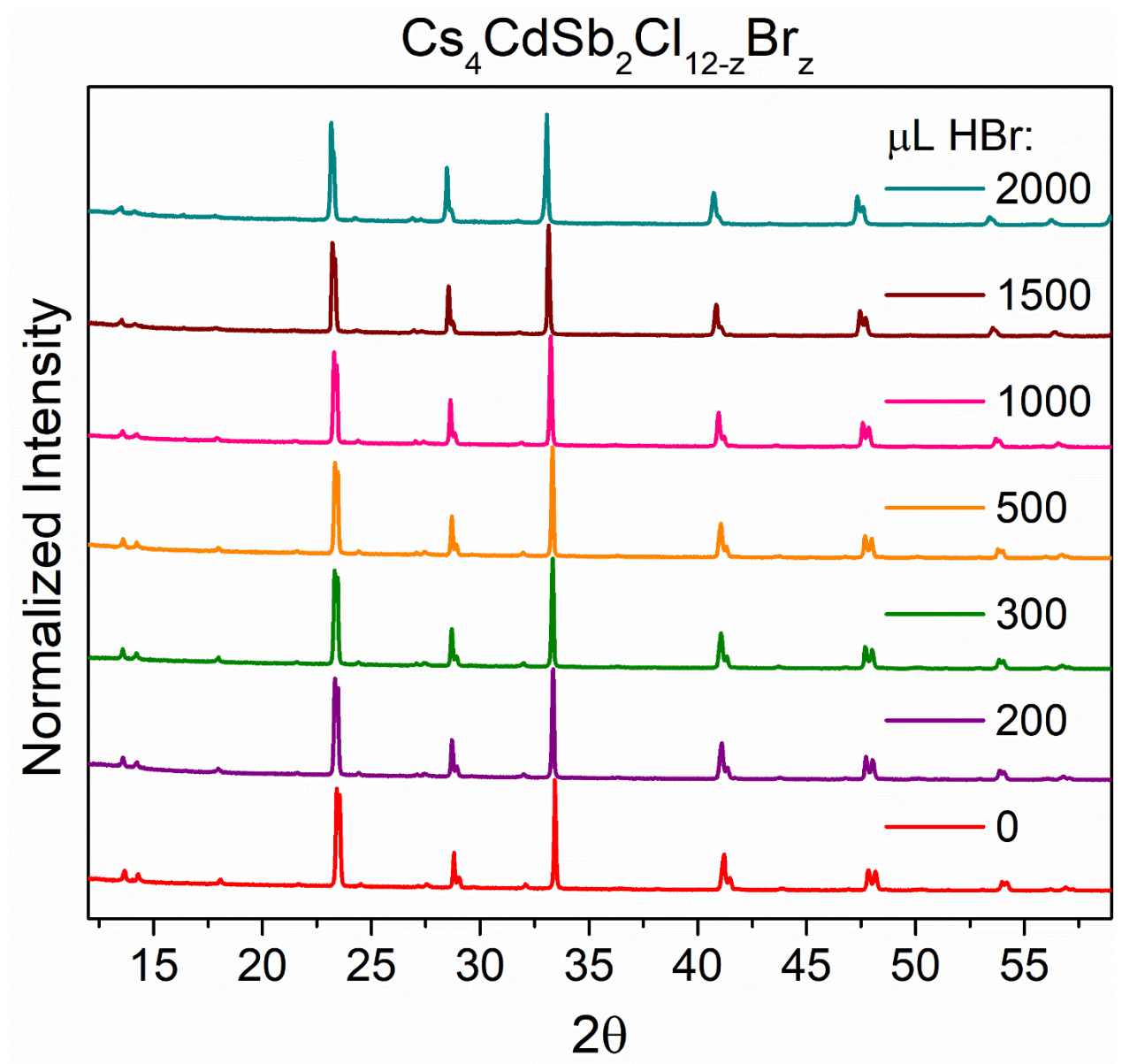

Figure S3. Stacked PXRD patterns of $\mathrm{Cs}_{4} \mathrm{CdSb}_{2} \mathrm{Cl}_{12-2} \mathrm{Br}_{z}$ specimens prepared from solutions containing varying amounts of $\operatorname{HBr}(a q)$. 


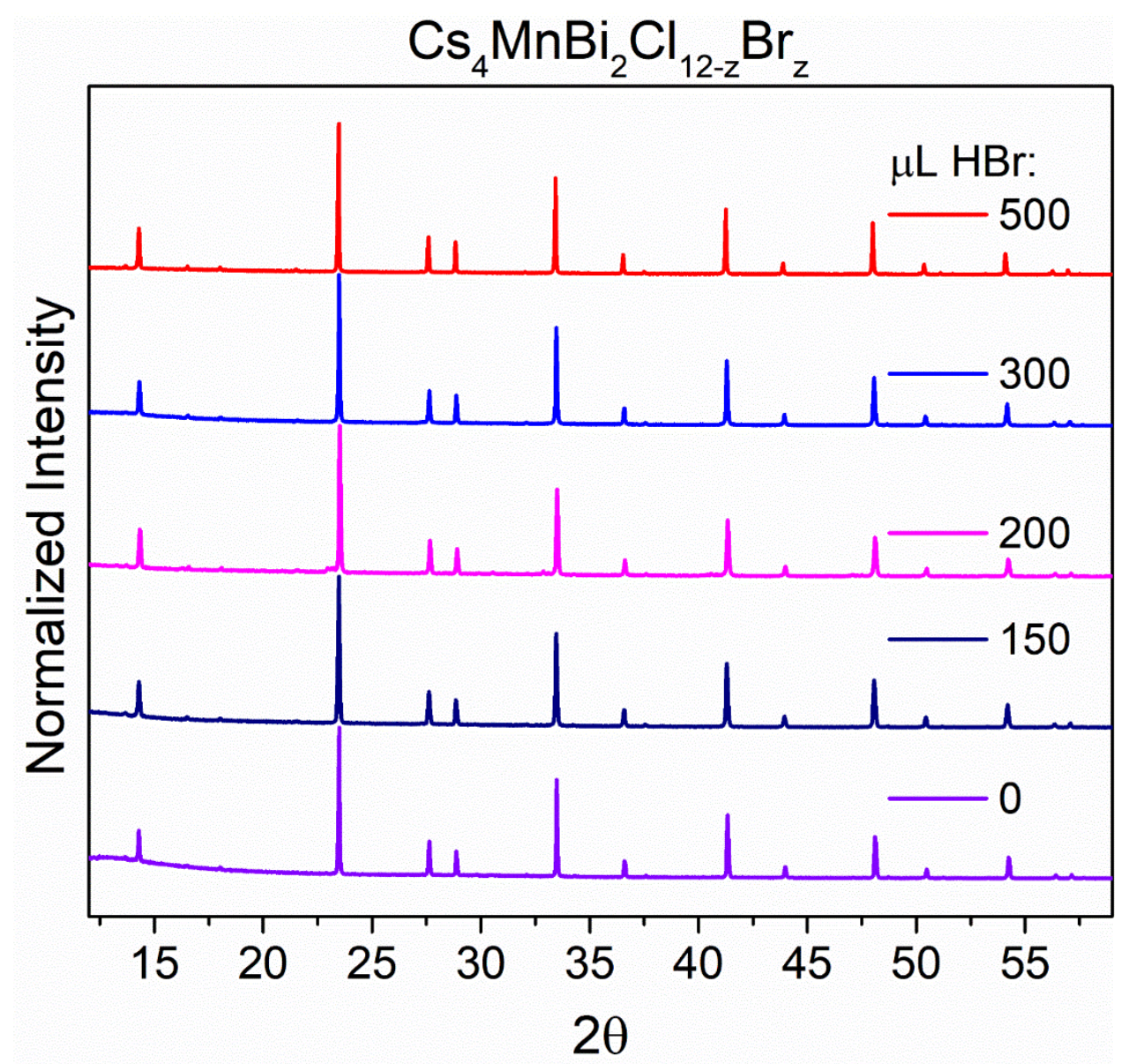

Figure S4. Stacked PXRD patterns of $\mathrm{Cs}_{4} \mathrm{MnBi}_{2} \mathrm{Cl}_{12-2} \mathrm{Br}_{z}$ specimens prepared from solutions containing varying amounts of $\operatorname{HBr}(a q)$. 


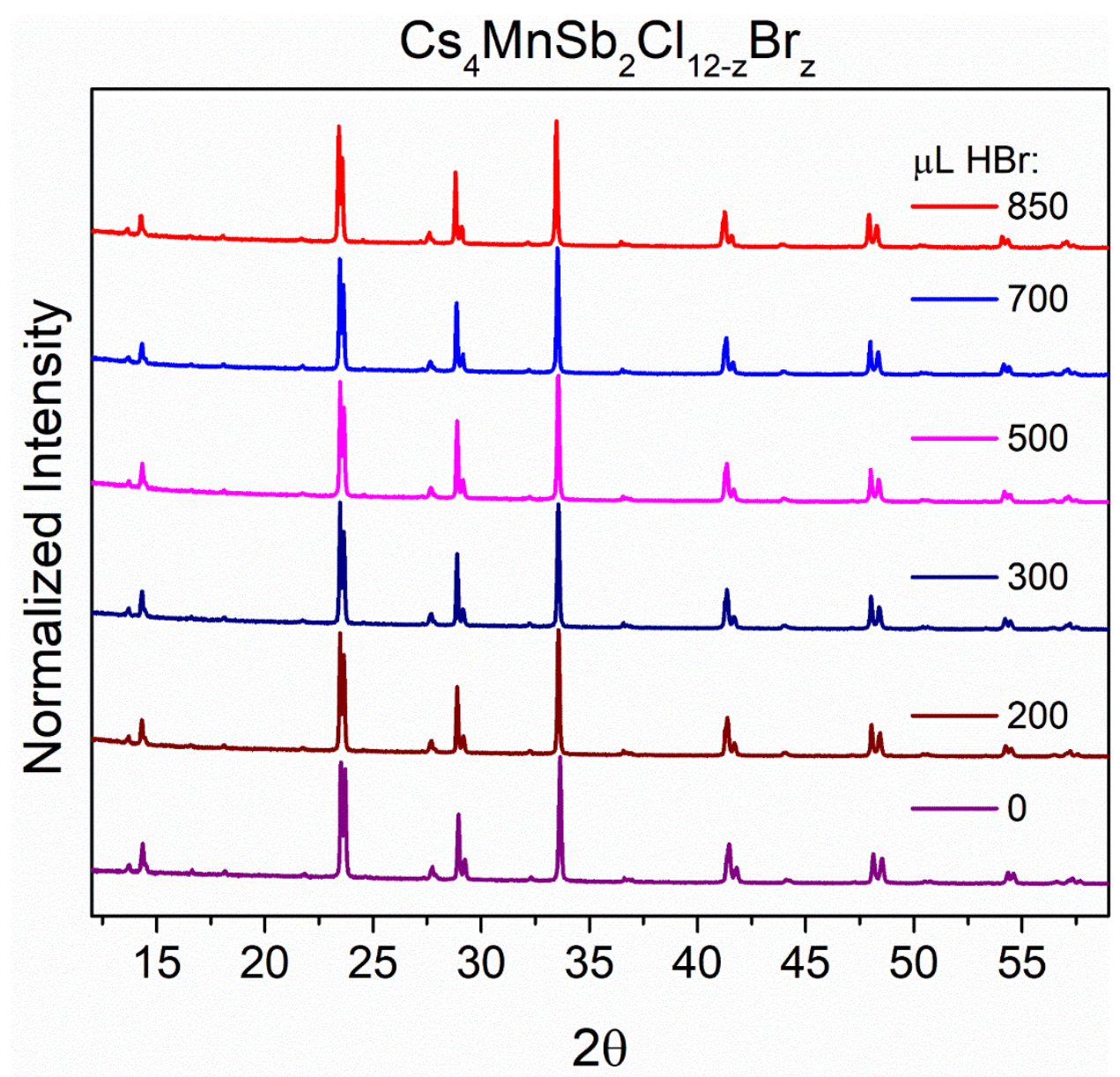

Figure S5. Stacked PXRD patterns of $\mathrm{Cs}_{4} \mathrm{MnSb}_{2} \mathrm{Cl}_{12-} \mathrm{Br}_{z}$ specimens prepared from solutions containing varying amounts of $\operatorname{HBr}(a q)$. 


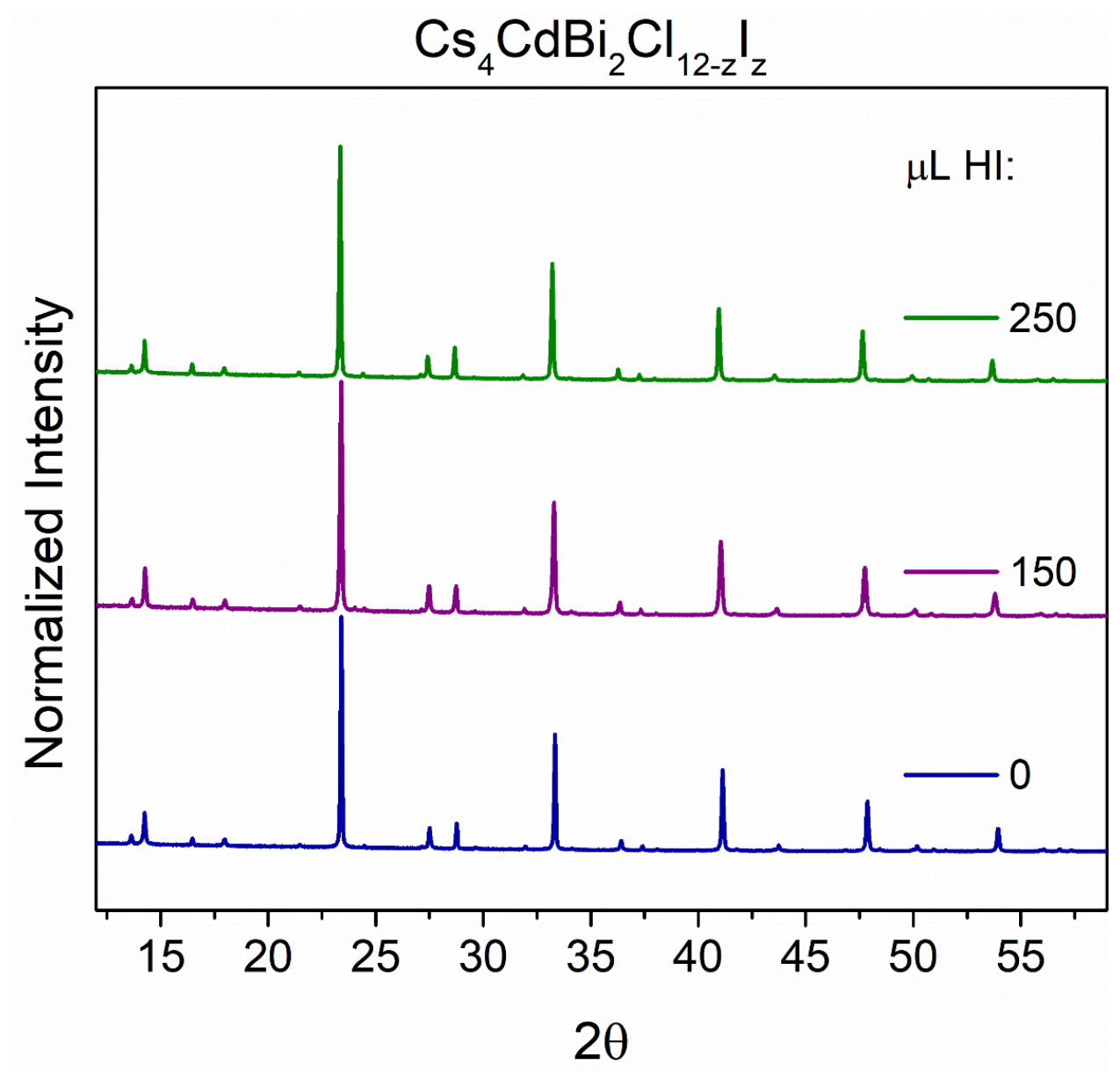

Figure S6. Stacked PXRD patterns of $\mathrm{Cs}_{4} \mathrm{CdBi}_{2} \mathrm{Cl}_{12-\mathrm{z}} \mathrm{I}_{\mathrm{z}}$ specimens prepared from solutions containing varying amounts of $\mathrm{HI}(a q)$. 


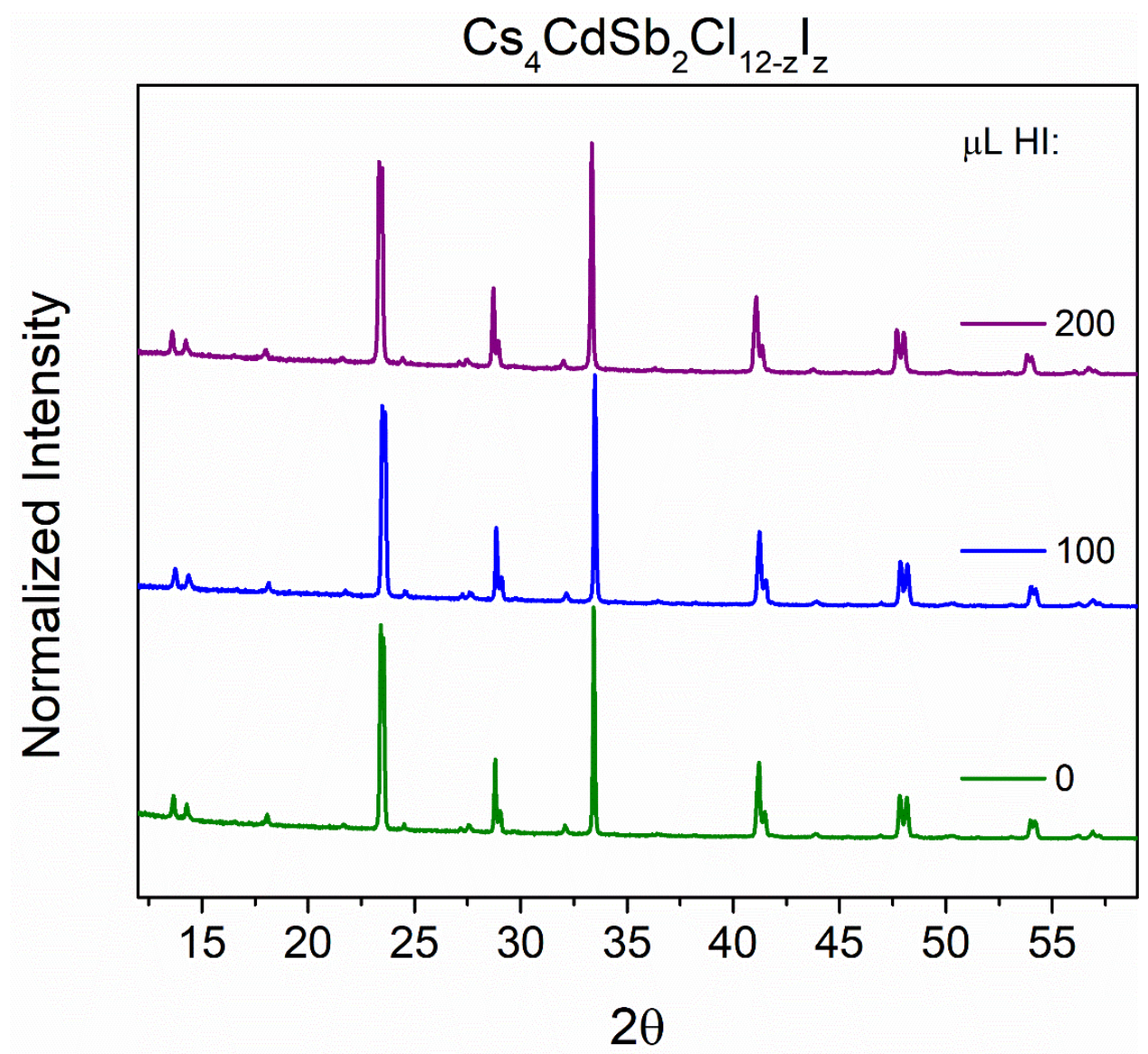

Figure S7. Stacked PXRD patterns of $\mathrm{Cs}_{4} \mathrm{CdSb}_{2} \mathrm{Cl}_{12-z} \mathrm{I}_{2}$ specimens prepared from solutions containing varying amounts of $\mathrm{HI}(a q)$. 


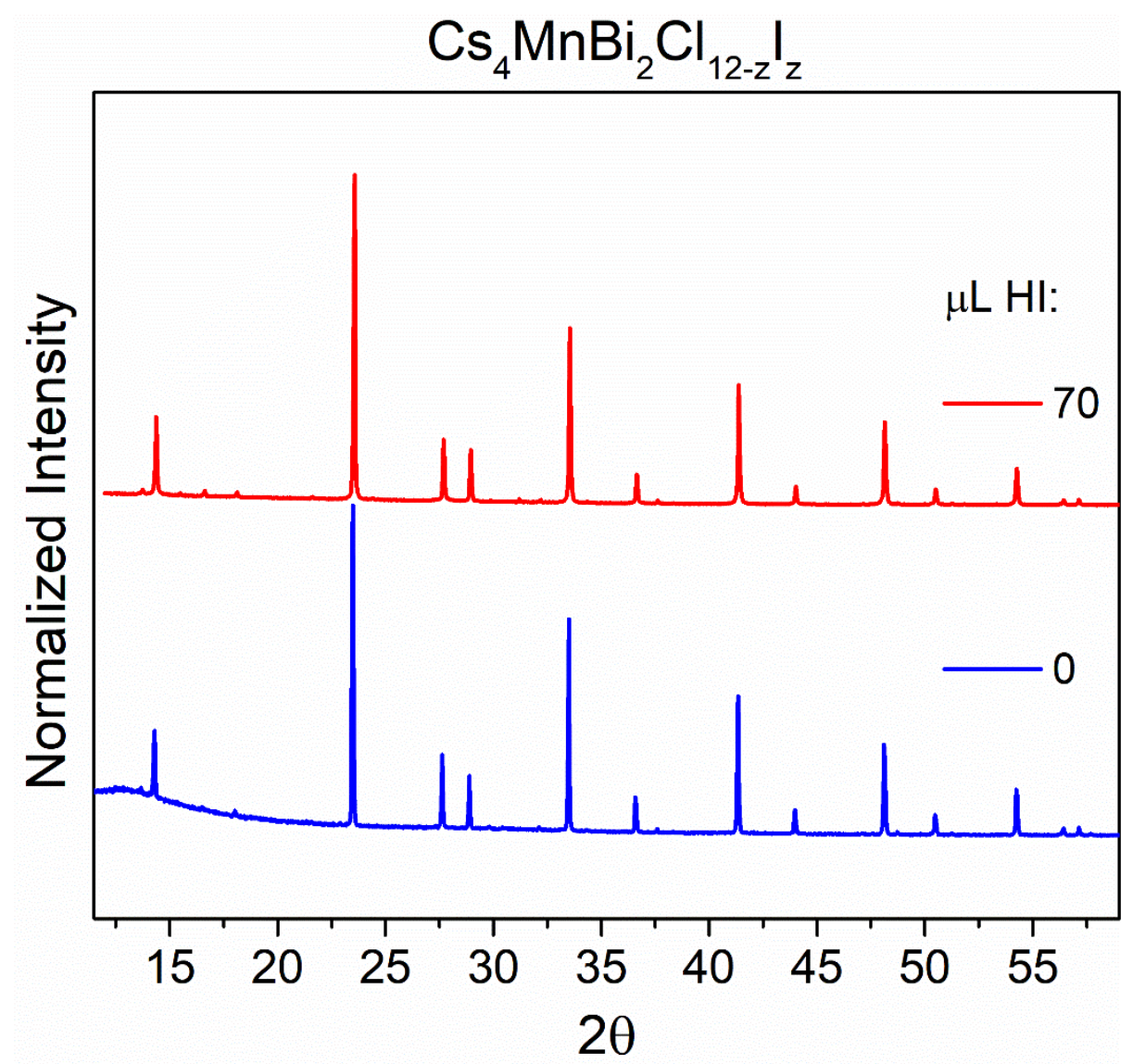

Figure S8. Stacked PXRD patterns of $\mathrm{Cs}_{4} \mathrm{MnBi}_{2} \mathrm{Cl}_{12-\mathrm{z}} \mathrm{I}_{\mathrm{z}}$ specimens prepared from solutions containing varying amounts of $\mathrm{HI}(a q)$. 


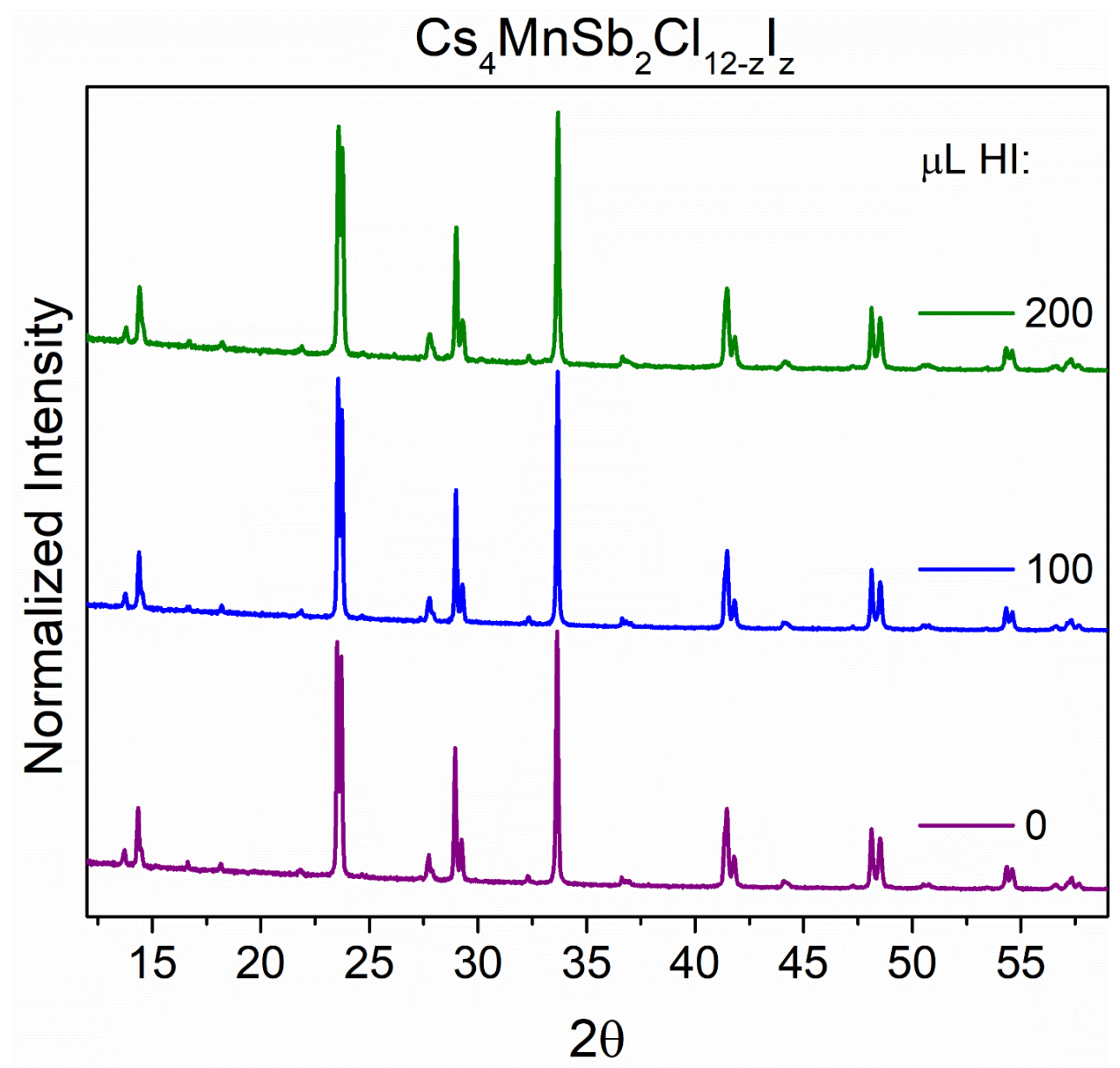

Figure S9. Stacked PXRD patterns of $\mathrm{Cs}_{4} \mathrm{MnSb}_{2} \mathrm{Cl}_{12-z} \mathrm{I}_{\mathrm{z}}$ specimens prepared from solutions containing varying amounts of $\mathrm{HI}(a q)$. 


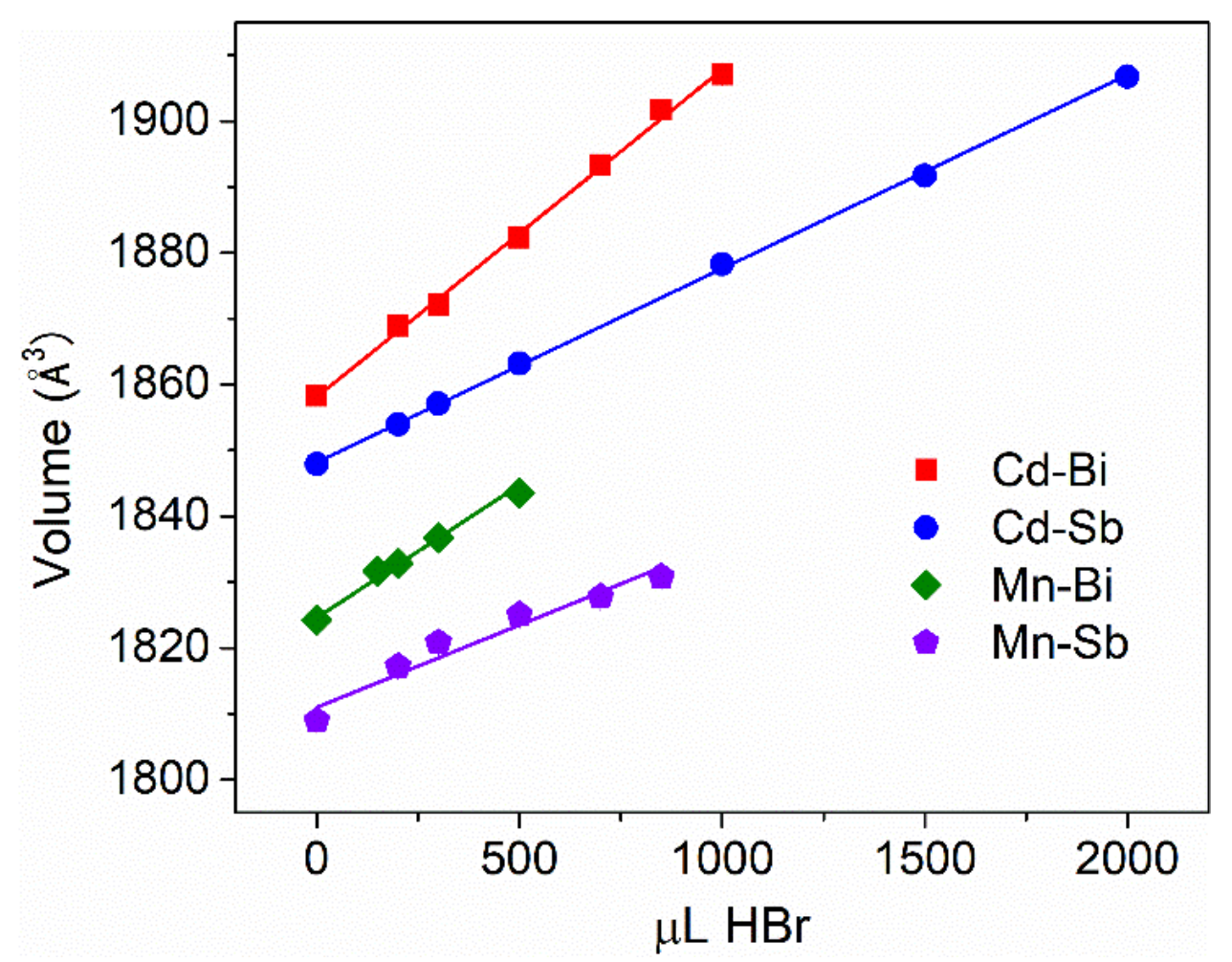

Figure S10. Lattice volume of all host compounds as increasing amounts of $\operatorname{HBr}(a q)$ are added to the reactions. 


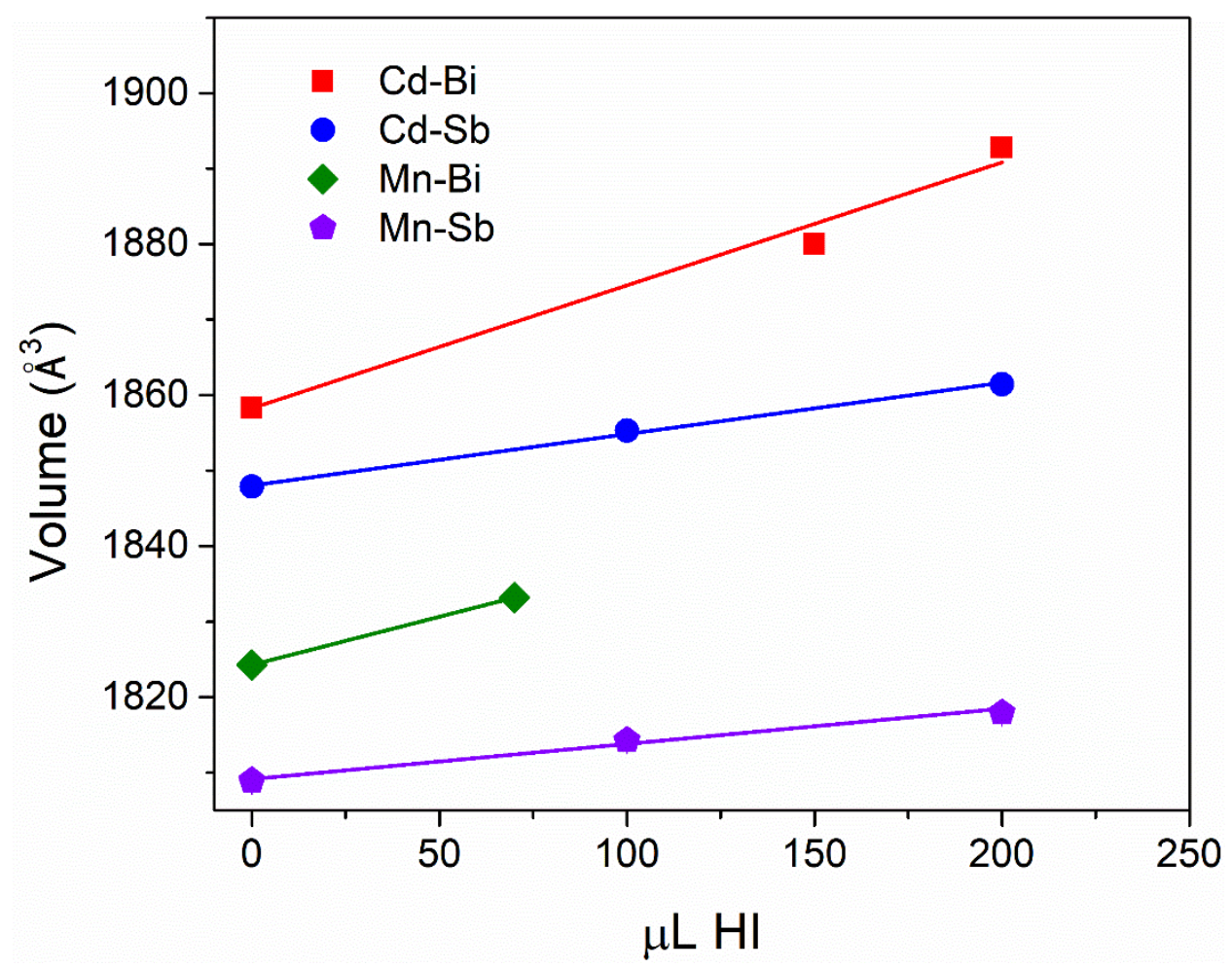

Figure S11. Lattice volume of all host compounds as increasing amounts of $\mathrm{HI}(a q)$ are added to the reactions. 

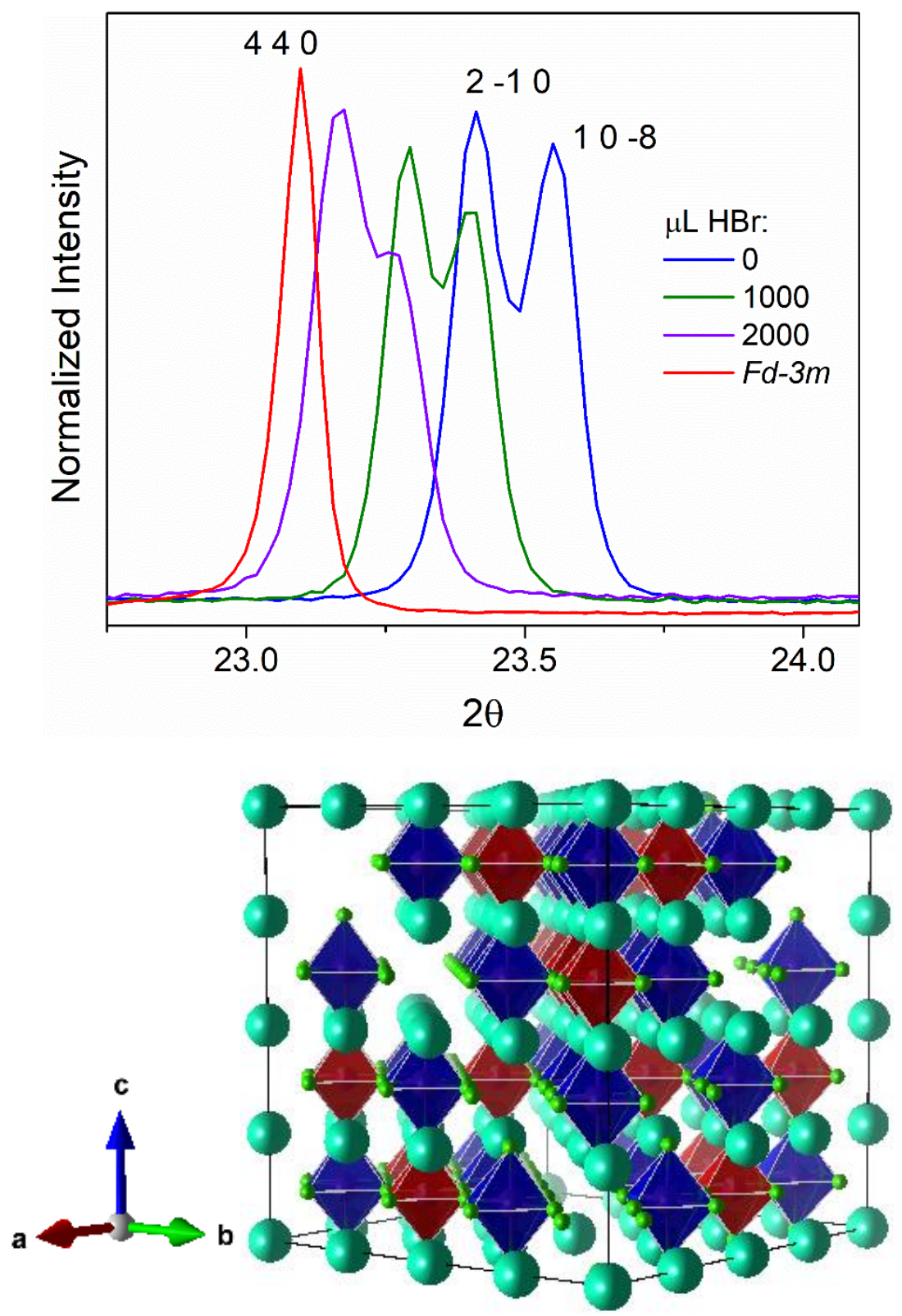

Figure S12. (Top) Peak splitting observed in PXRD patterns of $\mathrm{Cs}_{4} \mathrm{CdSb}_{2} \mathrm{Cl}_{12}$ (blue), as well as samples synthesized upon addition of $1000 \mu \mathrm{L}$ (green) and $2000 \mu \mathrm{L}$ (purple) of $\operatorname{HBr}(a q)$ to the reaction mixture prior to precipitation. For comparison, a simulated PXRD pattern, with $F d \overline{3} m$ symmetry (red), shows a single allowed reflection, (440). However, when a rhombohedral structure with $R \overline{3} m$ symmetry is adopted, this peak splits into

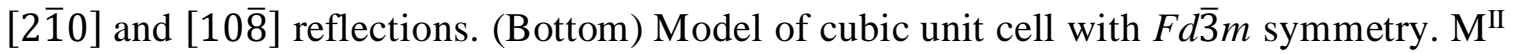
and $\mathrm{M}^{\mathrm{III}}$ are represented by red and blue octahedron, respectively. Cesium and chloride ions are represented by cyan and green spheres, respectively. 

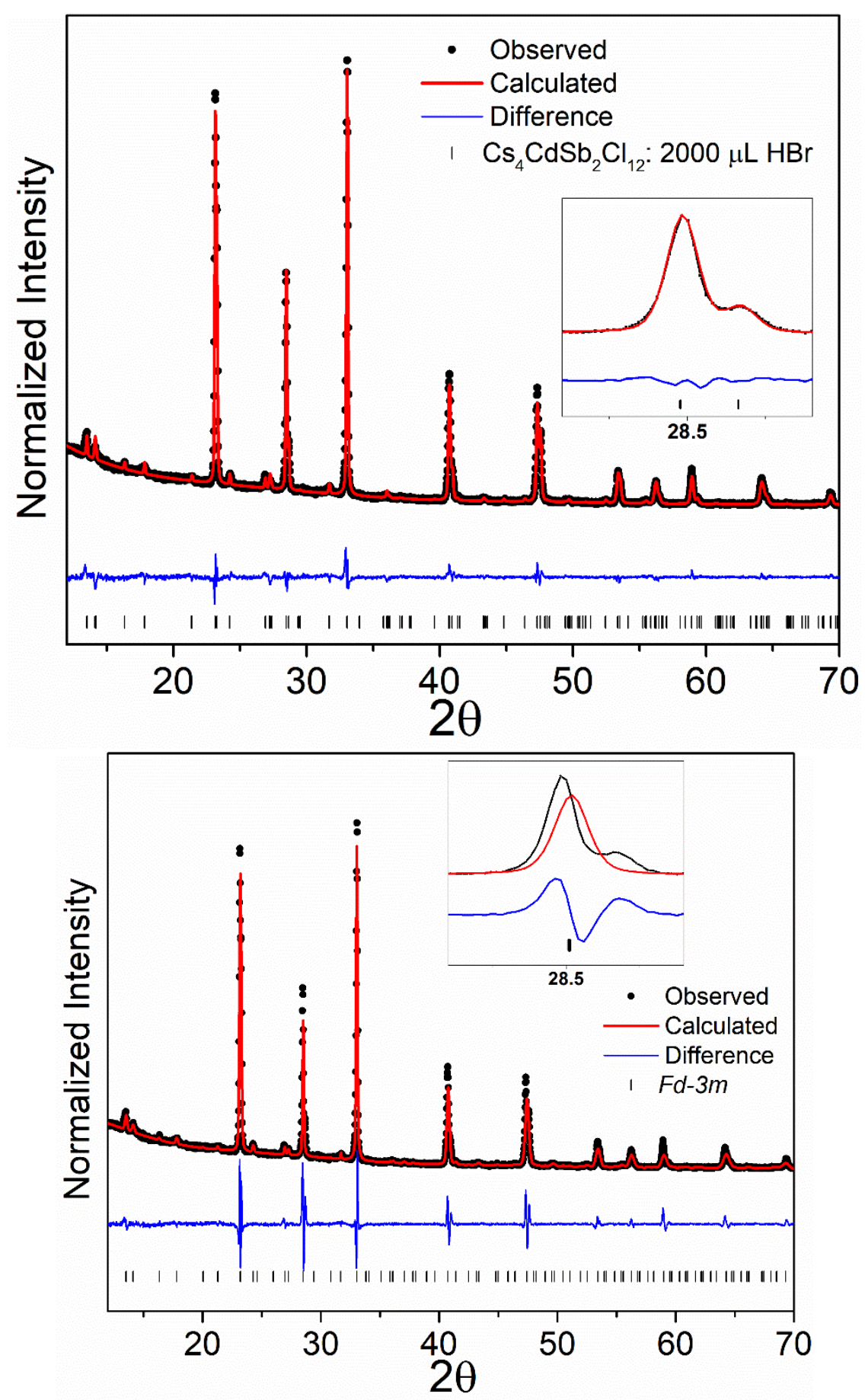

Figure S13. Rietveld refinement of $\mathrm{Cs}_{4} \mathrm{CdSb}_{2} \mathrm{Cl}_{12}: 2000 \mu \mathrm{L} \mathrm{HBr}$ in the $R \overline{3} m$ space group (top) and an attempted Pawley refinement in the $F d \overline{3} m$ space group (bottom). Inset of bottom image shows two peaks where only one reflection is allowed by the cubic structure. 


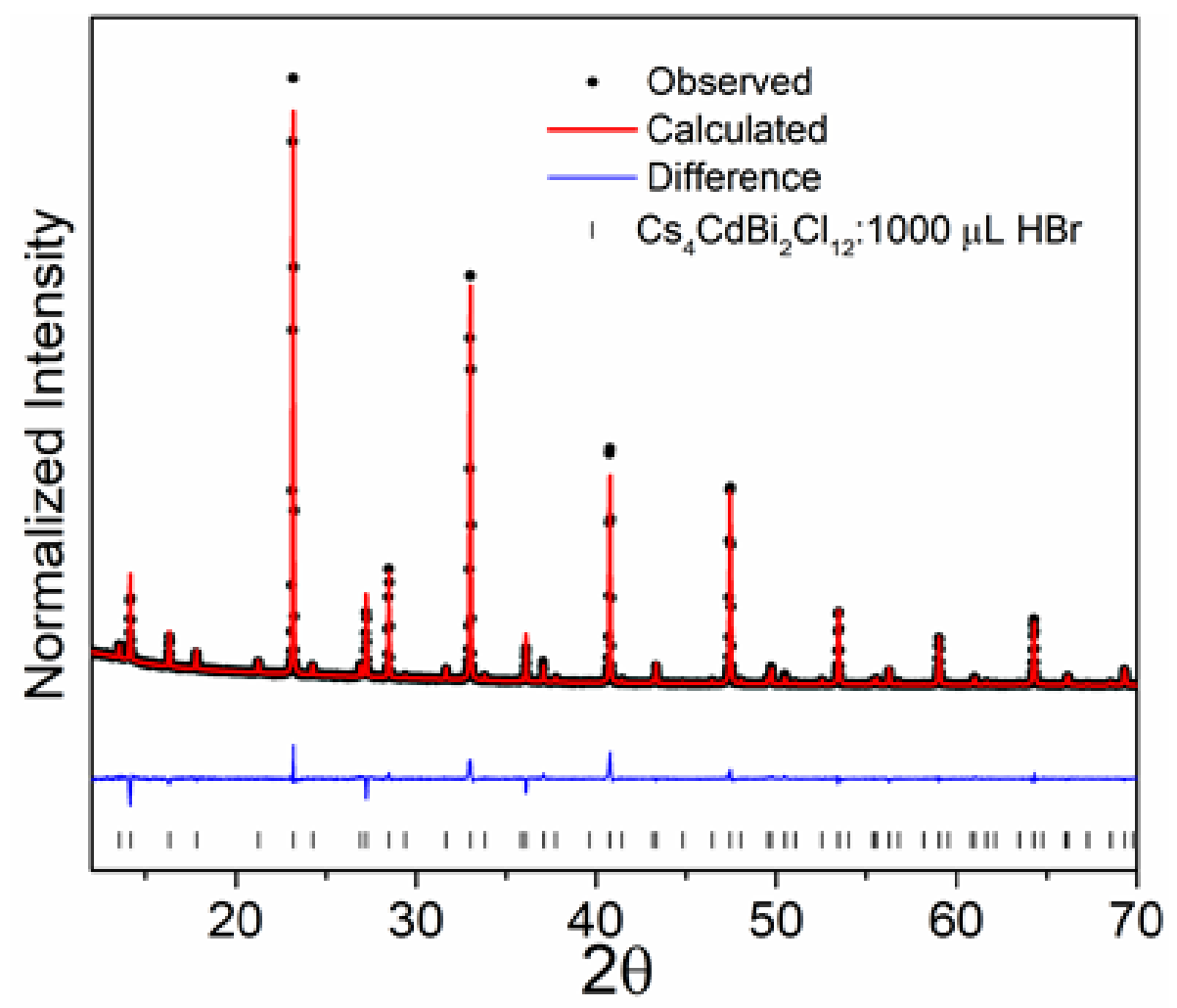

Figure S14. Rietveld refinement of $\mathrm{Cs}_{4} \mathrm{CdBi}_{2} \mathrm{Cl}_{12}: 1000 \mu \mathrm{L} \mathrm{HBr}$. 


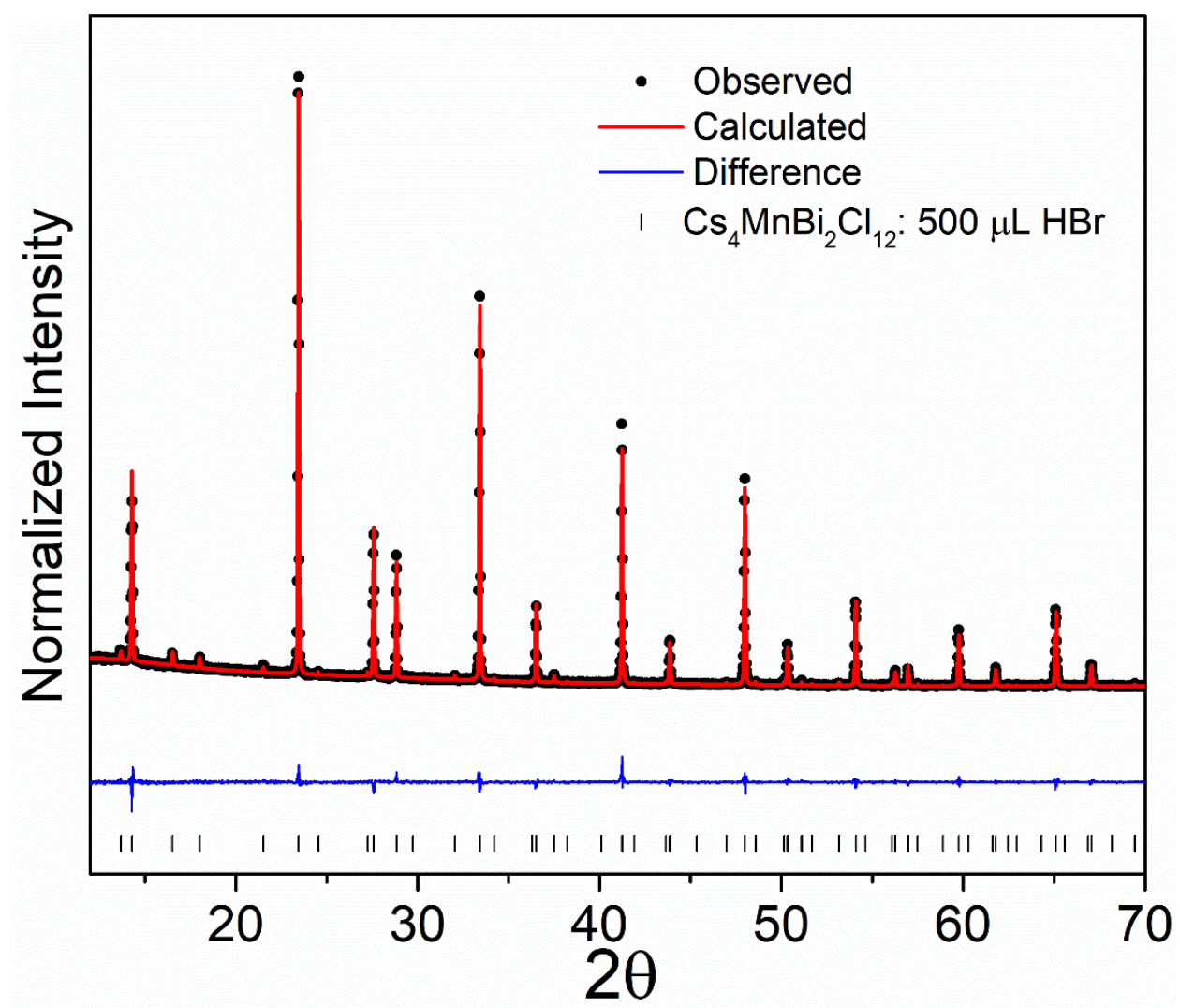

Figure S15. Rietveld refinement of $\mathrm{Cs}_{4} \mathrm{MnBi}_{2} \mathrm{Cl}_{12}: 500 \mu \mathrm{L} \mathrm{HBr}$. 


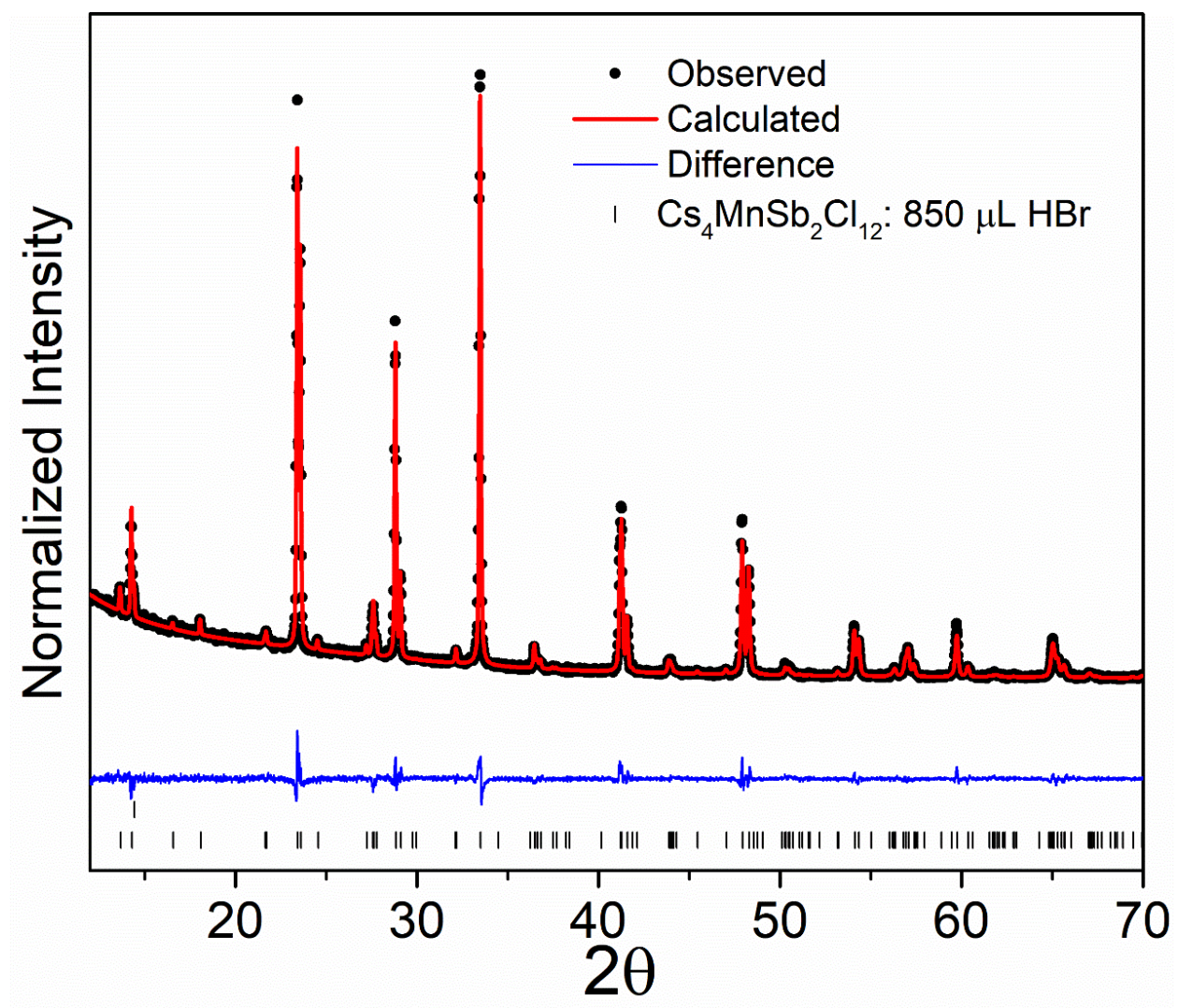

Figure S16. Rietveld refinement of $\mathrm{Cs}_{4} \mathrm{MnSb}_{2} \mathrm{Cl}_{12}: 850 \mu \mathrm{L} \mathrm{HBr}$. 


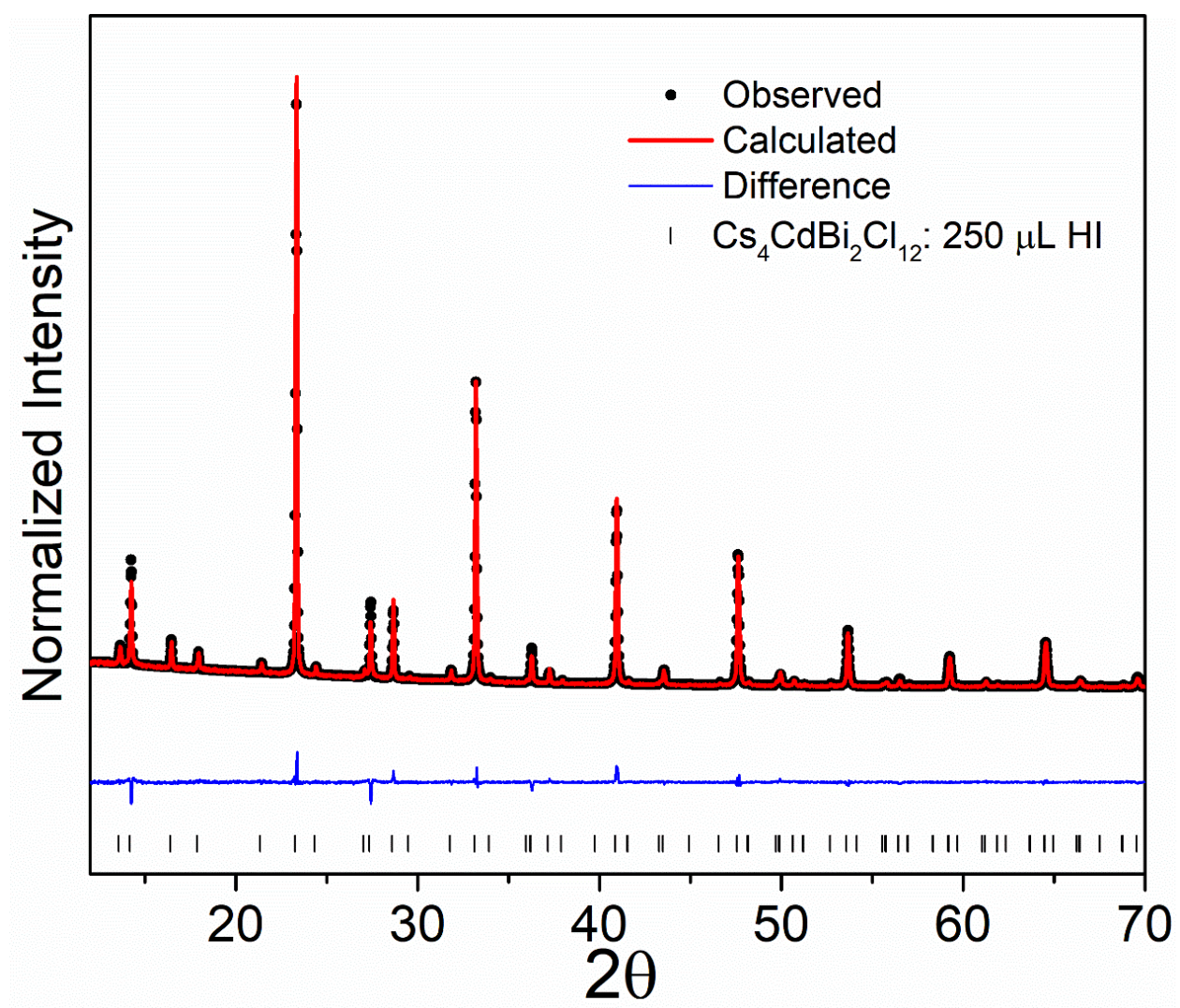

Figure S17. Rietveld refinement of $\mathrm{Cs}_{4} \mathrm{CdBi}_{2} \mathrm{Cl}_{12}: 250 \mu \mathrm{L} \mathrm{HI}$. 


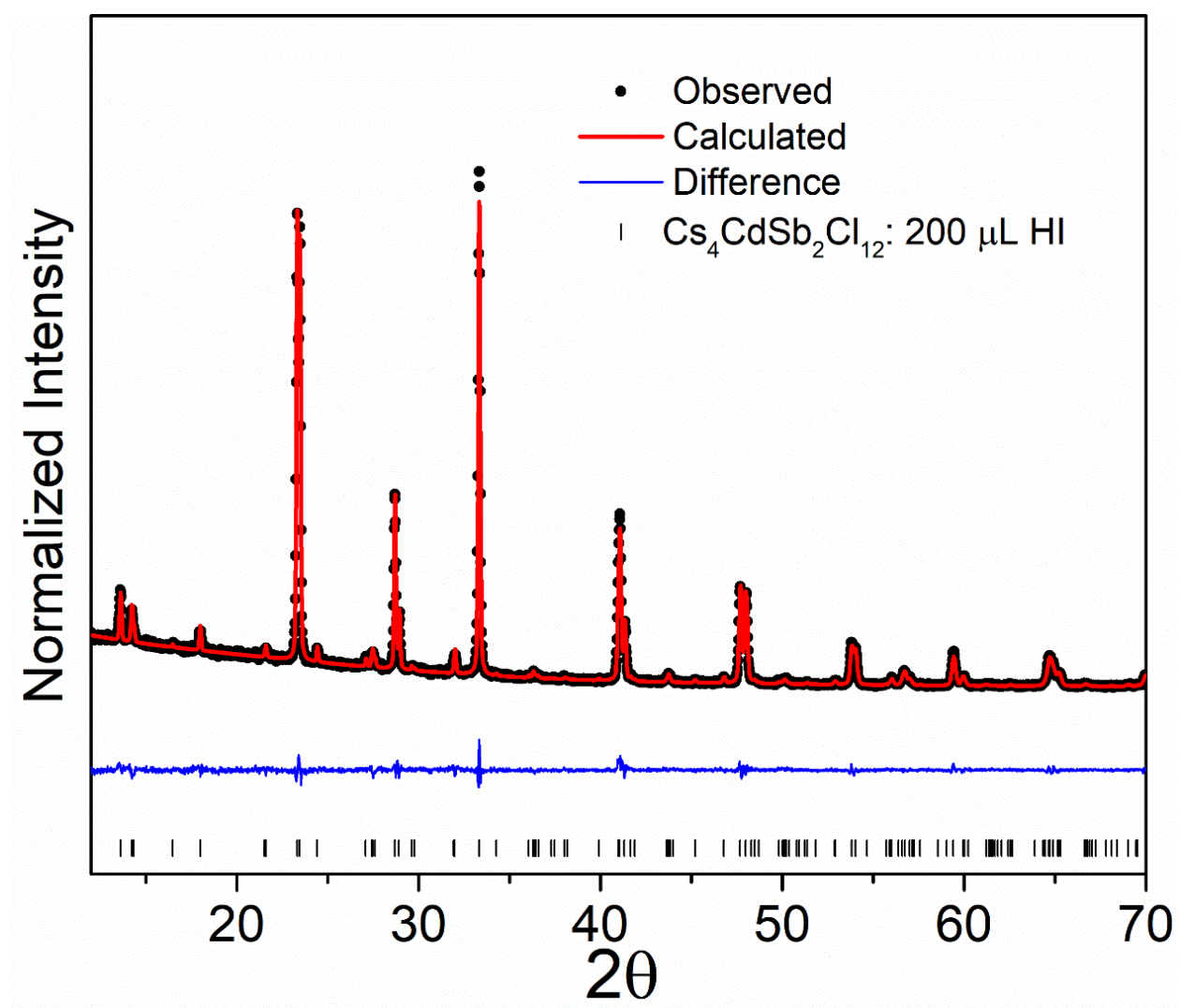

Figure S18. Rietveld refinement of $\mathrm{Cs}_{4} \mathrm{CdSb}_{2} \mathrm{Cl}_{12}: 200 \mu \mathrm{L} \mathrm{HI}$. 


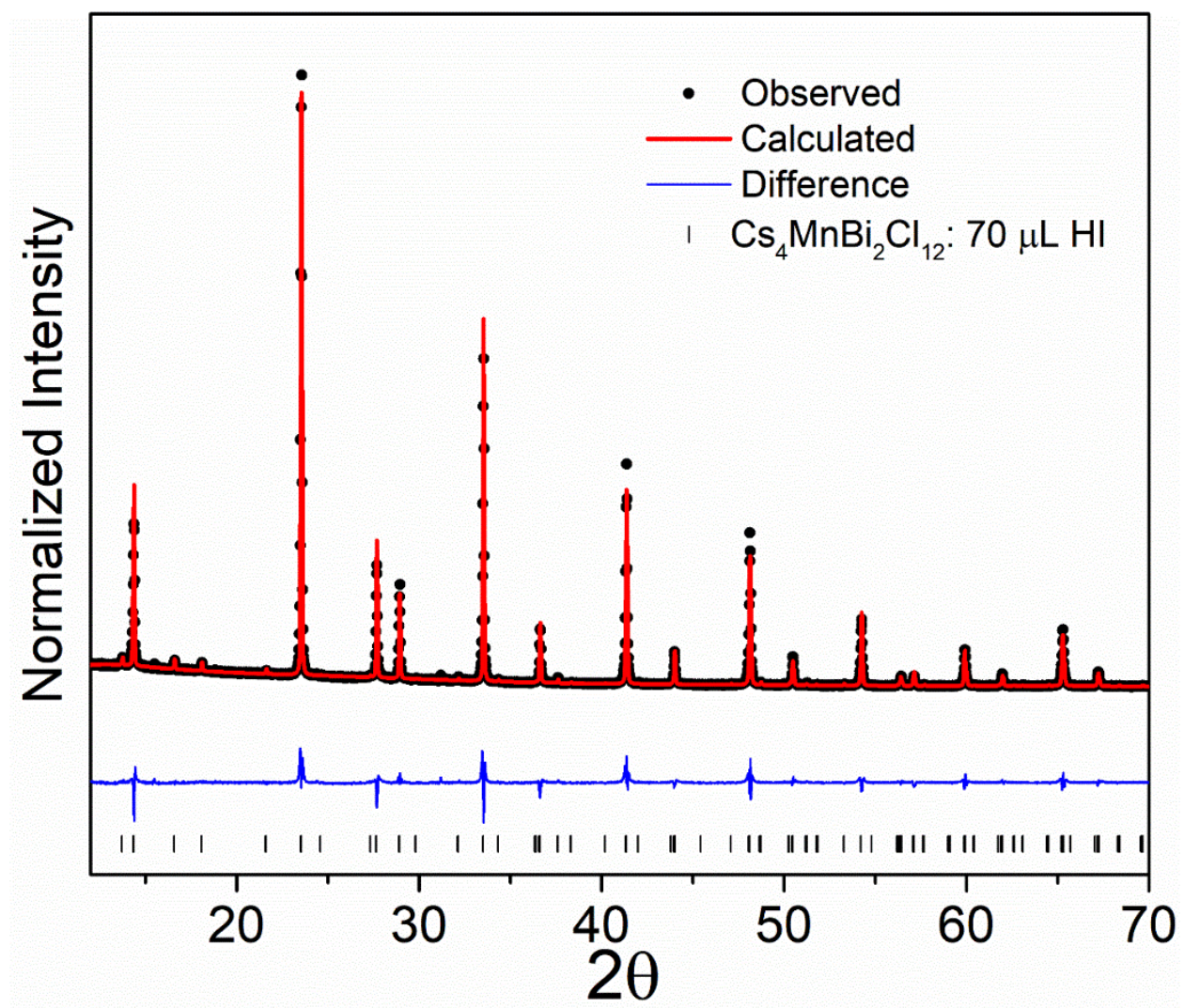

Figure S19. Rietveld refinement of $\mathrm{Cs}_{4} \mathrm{MnBi}_{2} \mathrm{Cl}_{12}: 70 \mu \mathrm{L} \mathrm{HI}$. 


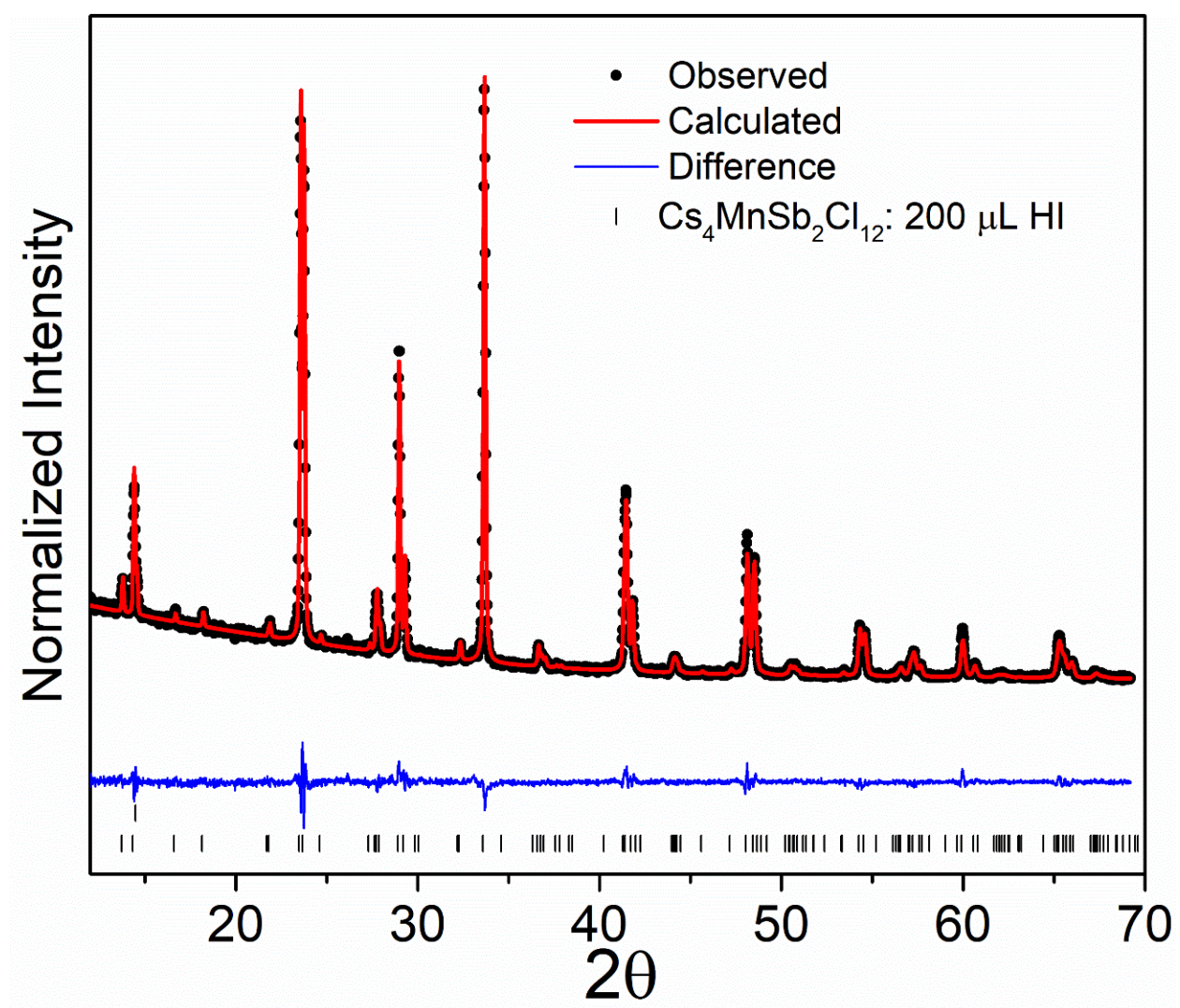

Figure S20. Rietveld refinement of $\mathrm{Cs}_{4} \mathrm{MnSb}_{2} \mathrm{Cl}_{12}: 200 \mu \mathrm{L} \mathrm{HI}$. 
Table S1. Summary of Rietveld refinement data for all hosts with maximum bromide and iodide substitutions. All scans were measured at $298 \mathrm{~K}$ from $10^{\circ}$ to $70^{\circ} 2 \theta$ with a step size of $0.02^{\circ} 2 \theta$ with 1 second per step under $1.5406 \AA \mathrm{Cu}$ radiation. All compounds have $R \overline{3} m H$ (\#166) space group symmetry.

\begin{tabular}{|c|c|c|c|c|}
\hline Compound & $\boldsymbol{a}(\AA)$ & $\boldsymbol{c}(\stackrel{\AA}{\mathbf{A}})$ & Volume $\left(\AA^{\mathbf{3}}\right)$ & $\mathbf{R}_{\boldsymbol{w}}$ \\
\hline $\mathrm{Cs}_{4} \mathrm{CdBi}_{2} \mathrm{Cl}_{12}: 1000 \mu \mathrm{L} \mathrm{HBr}$ & $7.6610(1)$ & $37.518(1)$ & $1907.0(1)$ & 7.722 \\
\hline $\mathrm{Cs}_{4} \mathrm{CdSb}_{2} \mathrm{Cl}_{12}: 2000 \mu \mathrm{L} \mathrm{HBr}$ & $7.6780(2)$ & $37.346(1)$ & $1906.7(1)$ & 7.111 \\
\hline $\mathrm{Cs}_{4} \mathrm{MnBi}_{2} \mathrm{Cl}_{12}: 500 \mu \mathrm{L} \mathrm{HBr}$ & $7.5745(1)$ & $37.102(1)$ & $1843.45(9)$ & 5.735 \\
\hline $\mathrm{Cs}_{4} \mathrm{MnSb}_{2} \mathrm{Cl}_{12}: 850 \mu \mathrm{L} \mathrm{HBr}$ & $7.5829(1)$ & $36.7653(8)$ & $1830.80(6)$ & 6.031 \\
\hline $\mathrm{Cs}_{4} \mathrm{CdBi}_{2} \mathrm{Cl}_{12}: 250 \mu \mathrm{L} \mathrm{HI}$ & $7.6426(3)$ & $37.421(3)$ & $1892.8(2)$ & 6.258 \\
\hline $\mathrm{Cs}_{4} \mathrm{CdSb}_{2} \mathrm{Cl}_{12}: 200 \mu \mathrm{L} \mathrm{HI}$ & $7.6214(1)$ & $37.0034(8)$ & $1861.42(6)$ & 5.662 \\
\hline $\mathrm{Cs}_{4} 4 \mathrm{MnBi}_{2} \mathrm{Cl}_{12}: 70 \mu \mathrm{L} \mathrm{HI}$ & $7.5560(6)$ & $37.036(6)$ & $1833.2(4)$ & 6.232 \\
\hline $\mathrm{Cs}_{4} \mathrm{MnSb}_{2} \mathrm{Cl}_{12}: 200 \mu \mathrm{L} \mathrm{HI}$ & $7.5677(1)$ & $36.654(1)$ & $1817.90(8)$ & 5.473 \\
\hline
\end{tabular}


Table S2. Summary of the relationship of the volume of $\mathrm{HBr}$ added to the solution, the total refined bromide incorporation, and unit cell volume.

\begin{tabular}{|c|c|c|c|}
\hline & Volume $\mathrm{HBr}$ added $(\mu \mathrm{L})$ & Refined total \% Br & Volume $\left(\AA^{3}\right)$ \\
\hline \multirow[t]{7}{*}{$\mathrm{Cs}_{4} \mathrm{CdBi}_{2} \mathrm{Cl}_{12}$} & 0 & $0(2)$ & $1858.30(6)$ \\
\hline & 200 & $7(2)$ & $1868.80(6)$ \\
\hline & 300 & $6(2)$ & $1872.10(6)$ \\
\hline & 500 & $10(2)$ & $1882.30(6)$ \\
\hline & 700 & $19(2)$ & $1893.30(6)$ \\
\hline & 850 & $23(2)$ & $1901.7(1)$ \\
\hline & 1000 & $26(2)$ & $1907.0(1)$ \\
\hline \multirow[t]{7}{*}{$\mathrm{Cs}_{4} \mathrm{CdSb}_{2} \mathrm{Cl}_{12}$} & 0 & $0(2)$ & $1847.87(5)$ \\
\hline & 200 & $7(1)$ & $1853.91(6)$ \\
\hline & 300 & $9(1)$ & $1857.09(6)$ \\
\hline & 500 & $12(1)$ & $1863.18(6)$ \\
\hline & 1000 & $19(1)$ & $1878.25(6)$ \\
\hline & 1500 & $25(2)$ & 1891.71(7) \\
\hline & 2000 & $29(2)$ & $1906.7(1)$ \\
\hline \multirow[t]{5}{*}{$\mathrm{Cs}_{4} \mathrm{MnBi}_{2} \mathrm{Cl}_{12}$} & 0 & $0(2)$ & $1824.27(3)$ \\
\hline & 150 & $6(2)$ & $1831.66(5)$ \\
\hline & 200 & $7(2)$ & $1832.86(5)$ \\
\hline & 300 & $8(2)$ & $1836.71(4)$ \\
\hline & 500 & $11(2)$ & $1843.45(9)$ \\
\hline \multirow[t]{6}{*}{$\mathrm{Cs}_{4} \mathrm{MnSb}_{2} \mathrm{Cl}_{12}$} & 0 & $0(2)$ & $1808.92(5)$ \\
\hline & 200 & $6(1)$ & $1817.21(6)$ \\
\hline & 300 & $8(2)$ & $1820.80(6)$ \\
\hline & 500 & $12(2)$ & $1825.03(6)$ \\
\hline & 700 & $13(2)$ & $1827.71(6)$ \\
\hline & 850 & $15(2)$ & $1830.80(6)$ \\
\hline
\end{tabular}

Table S3. Summary of the relationship of the volume of HI added to the solution, the total refined iodide incorporation, and unit cell volume.

\begin{tabular}{|c|c|c|c|}
\hline & Volume HI added $(\mu \mathrm{L})$ & Refined total \% I & Volume $\left(\AA^{3}\right)$ \\
\hline $\mathrm{Cs}_{4} \mathrm{CdBi}_{2} \mathrm{Cl}_{12}$ & 0 & $0(2)$ & $1858.30(6)$ \\
\hline & 150 & $5.0(7)$ & $1880.0(2)$ \\
\hline & 250 & $5.8(6)$ & $1892.8(2)$ \\
\hline $\mathrm{Cs}_{4} \mathrm{CdSb}_{2} \mathrm{Cl}_{12}$ & 0 & $0(2)$ & $1847.87(5)$ \\
\hline & 100 & $2.7(6)$ & $1855.28(6)$ \\
\hline $\mathrm{Cs}_{4} \mathrm{MnBi}_{2} \mathrm{Cl}_{12}$ & 200 & $3.8(5)$ & $1861.42(6)$ \\
\hline & 0 & $0(2)$ & $1824.27(3)$ \\
\hline $\mathrm{Cs}_{4} \mathrm{MnSb}_{2} \mathrm{Cl}_{12}$ & 70 & $1(1)$ & $1833.2(4)$ \\
\hline & 0 & $0(2)$ & $1808.92(5)$ \\
\hline & 100 & $2.0(6)$ & $1814.26(5)$ \\
\hline & 200 & $3(1)$ & $1817.90(8)$ \\
\hline
\end{tabular}


Table S4. Lattice parameters of the mono-halide parent structures of the determined impurity phases. All values are for room temperature/ambient pressure structures from the literature and/or the ICSD. ${ }^{1-4}$ The impurity phases formed upon excessive halide substitution into the VOQP phases. Lattice parameters are determined by Pawley refinements and can be compared to the mono-halide parent structures.

\begin{tabular}{|c|c|c|c|c|c|c|c|}
\hline \multicolumn{4}{|c|}{ Mono-halide parent structures: } & & & & \\
\hline Phases: & $a(\AA)$ & $b(\AA)$ & $c(\AA)$ & Ref. & & & \\
\hline $\mathrm{Cs}_{3} \mathrm{Bi}_{2} \mathrm{Br}_{9}$ & 7.972 & 7.972 & 9.867 & 1 & & & \\
\hline $\mathrm{Cs}_{3} \mathrm{Sb}_{2} \mathrm{Br}_{9}$ & 7.93 & 7.93 & 9.716 & 1 & & & \\
\hline $\mathrm{Cs}_{3} \mathrm{Bi}_{2} \mathrm{I}_{9}$ & 8.404 & 8.404 & 21.183 & 1 & & & \\
\hline $\mathrm{Cs}_{3} \mathrm{Sb}_{2} \mathrm{I}_{9}$ & 8.349 & 8.349 & 20.936 & 1 & & & \\
\hline $\mathrm{CsMnCl}_{3} \cdot\left(\mathrm{H}_{2} \mathrm{O}\right)_{2}$ & 9.031 & 7.264 & 11.431 & 1 & & & \\
\hline $\mathrm{Cs}_{4} \mathrm{CdBi}_{2} \mathrm{Cl}_{12}$ & 7.596 & 7.596 & 37.185 & 2 & & & \\
\hline $\mathrm{Cs}_{4} \mathrm{CdSb}_{2} \mathrm{Cl}_{12}$ & 7.603 & 7.603 & 36.916 & 3 & & & \\
\hline $\mathrm{Cs}_{4} \mathrm{MnBi}_{2} \mathrm{Cl}_{12}$ & 7.551 & 7.551 & 36.942 & 2 & & & \\
\hline $\mathrm{Cs}_{4} \mathrm{MnSb}_{2} \mathrm{Cl}_{12}$ & 7.554 & 7.554 & 36.601 & 4 & & & \\
\hline \multicolumn{4}{|c|}{$\mathrm{Cs}_{4} \mathrm{CdBi}_{2} \mathrm{Cl}_{12}-\mathrm{Br}_{\mathrm{z}}$} & \multicolumn{4}{|c|}{$\mathrm{Cs}_{4} \mathrm{CdBi}_{2} \mathrm{Cl}_{12-\mathrm{z}} \mathrm{I}_{\mathrm{z}}$} \\
\hline Phases: & $a(\AA)$ & $b(\AA)$ & $c(\AA)$ & Phases: & $a(\AA)$ & $b(\AA)$ & $c(\AA)$ \\
\hline $\mathrm{Cs}_{4} \mathrm{CdBi}_{2} \mathrm{Cl}_{12-z} \mathrm{Br}_{\mathrm{z}}$ & 7.684 & 7.684 & 37.638 & $\mathrm{Cs}_{4} \mathrm{CdBi}_{2} \mathrm{Cl}_{12-\mathrm{z}} \mathrm{I}_{\mathrm{z}}$ & 7.639 & 7.639 & 37.432 \\
\hline $\mathrm{Cs}_{3} \mathrm{Bi}_{2} \mathrm{Br}_{9-2} \mathrm{Cl}_{\mathrm{z}}$ & 7.724 & 7.724 & 9.517 & $\mathrm{Cs}_{3} \mathrm{Bi}_{2} \mathrm{I}_{9-\mathrm{z}} \mathrm{Cl}_{\mathrm{z}}$ & 8.398 & 8.398 & 21.162 \\
\hline \multicolumn{4}{|c|}{$\mathrm{Cs}_{4} \mathrm{CdSb}_{2} \mathrm{Cl}_{12-z} \mathrm{Br}_{\mathrm{z}}$} & \multicolumn{4}{|c|}{$\mathrm{Cs}_{4} \mathrm{CdSb}_{2} \mathrm{Cl}_{12-z} \mathrm{I}_{\mathrm{z}}$} \\
\hline Phases: & $a(\AA)$ & $b(\AA)$ & $c(\AA)$ & Phases: & $a(\AA)$ & $b(\AA)$ & $c(\AA)$ \\
\hline $\mathrm{Cs}_{4} \mathrm{CdSb}_{2} \mathrm{Cl}_{12-\mathrm{z}} \mathrm{Br}_{\mathrm{z}}$ & 7.688 & 7.688 & 37.389 & $\mathrm{Cs}_{4} \mathrm{CdSb}_{2} \mathrm{Cl}_{12-\mathrm{z}} \mathrm{I}_{\mathrm{z}}$ & 7.629 & 7.629 & 37.023 \\
\hline $\mathrm{Cs}_{3} \mathrm{Sb}_{2} \mathrm{Br}_{9-2} \mathrm{Cl}_{z}$ & 7.731 & 7.731 & 9.491 & $\mathrm{Cs}_{3} \mathrm{Sb}_{2} \mathrm{I}_{9-\mathrm{z}} \mathrm{Cl}_{\mathrm{z}}$ & 8.367 & 8.367 & 20.936 \\
\hline \multicolumn{4}{|c|}{$\mathrm{Cs}_{4} \mathrm{MnBi}_{2} \mathrm{Cl}_{12-z} \mathrm{Br}_{\mathrm{z}}$} & \multicolumn{4}{|c|}{$\mathrm{Cs}_{4} \mathrm{MnBi}_{2} \mathrm{Cl}_{12-z} \mathrm{I}_{\mathrm{z}}$} \\
\hline Phases: & $a(\AA)$ & $b(\AA)$ & $c(\AA)$ & Phases: & $a(\AA)$ & $b(\AA)$ & $c(\AA)$ \\
\hline $\mathrm{Cs}_{3} \mathrm{Bi}_{2} \mathrm{Br}_{-z} \mathrm{Cl}_{z}$ & 7.724 & 7.724 & 9.517 & $\mathrm{Cs}_{3} \mathrm{Bi}_{2} \mathrm{Cl}_{9-\mathrm{z}} \mathrm{I}_{\mathrm{z}}$ & 7.727 & 7.727 & 9.507 \\
\hline $\mathrm{CsMnCl}_{3} \cdot\left(\mathrm{H}_{2} \mathrm{O}\right)_{2}$ & 9.079 & 7.298 & 11.476 & $\mathrm{CsMnCl}_{3} \cdot\left(\mathrm{H}_{2} \mathrm{O}\right)_{2}$ & 9.077 & 7.296 & 11.476 \\
\hline \multicolumn{4}{|c|}{$\mathrm{Cs}_{4} \mathrm{MnSb}_{2} \mathrm{Cl}_{12-\mathrm{z}} \mathrm{Br}_{\mathrm{z}}$} & \multicolumn{4}{|c|}{$\mathrm{Cs}_{4} \mathrm{MnSb}_{2} \mathrm{Cl}_{12-z \mathrm{I}_{\mathrm{z}}}$} \\
\hline Phases: & $a(\AA)$ & $b(\AA)$ & $c(\AA)$ & Phases: & $a(\AA)$ & $b(\AA)$ & $c(\AA)$ \\
\hline $\mathrm{Cs}_{4} \mathrm{MnSb}_{2} \mathrm{Cl}_{12-2} \mathrm{Br}_{\mathrm{z}}$ & 7.586 & 7.586 & 36.78 & $\mathrm{Cs}_{4} \mathrm{MnSb}_{2} \mathrm{Cl}_{12-\mathrm{z}} \mathrm{I}_{\mathrm{z}}$ & 7.567 & 7.567 & 36.649 \\
\hline $\mathrm{Cs}_{3} \mathrm{Sb}_{2} \mathrm{Br}_{9-2} \mathrm{Cl}_{\mathrm{z}}$ & 7.691 & 7.691 & 9.396 & $\mathrm{Cs}_{3} \mathrm{Sb}_{2} \mathrm{I}_{9-\mathrm{z}} \mathrm{Cl}_{\mathrm{z}}$ & 8.343 & 8.343 & 20.892 \\
\hline
\end{tabular}



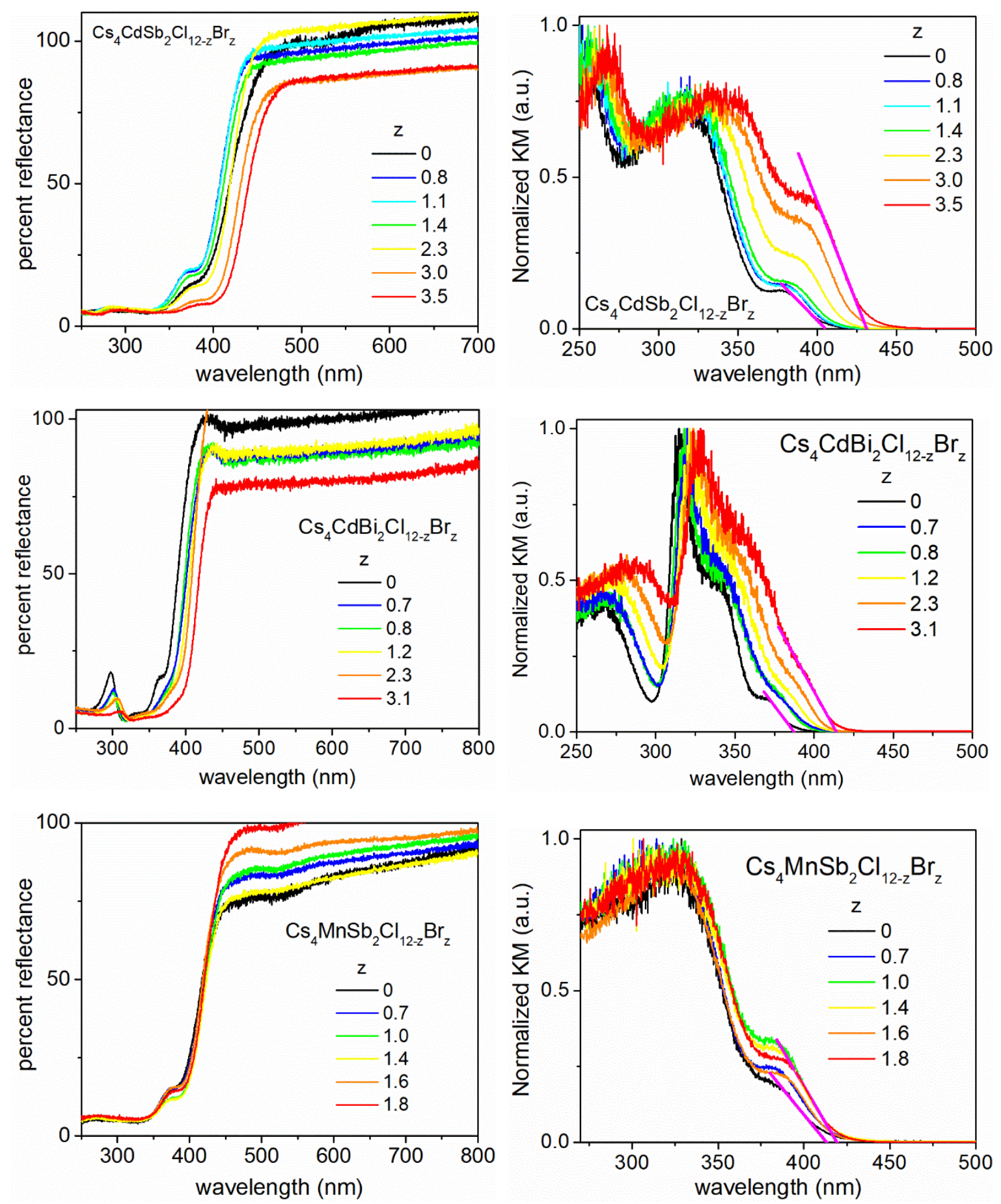

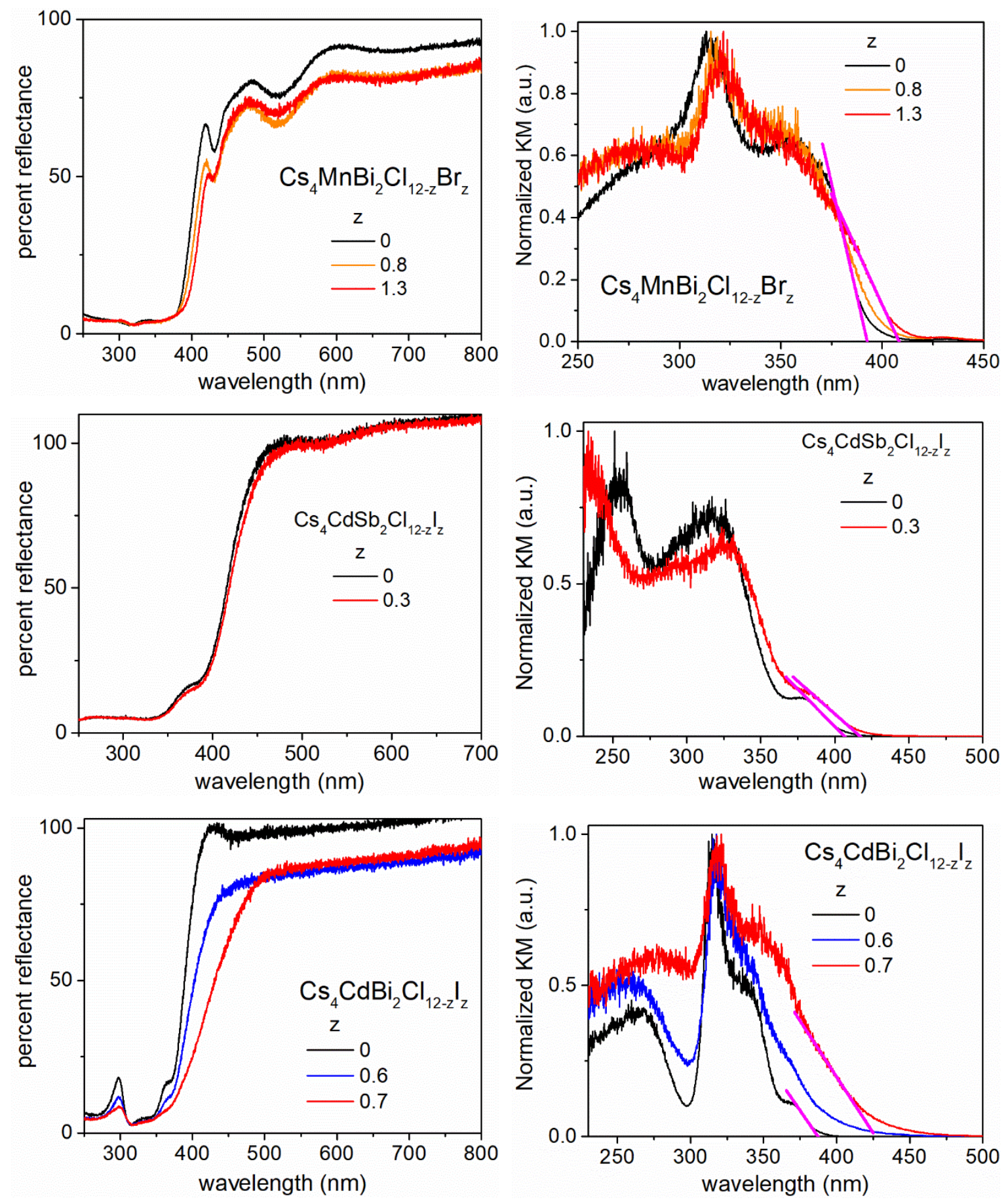

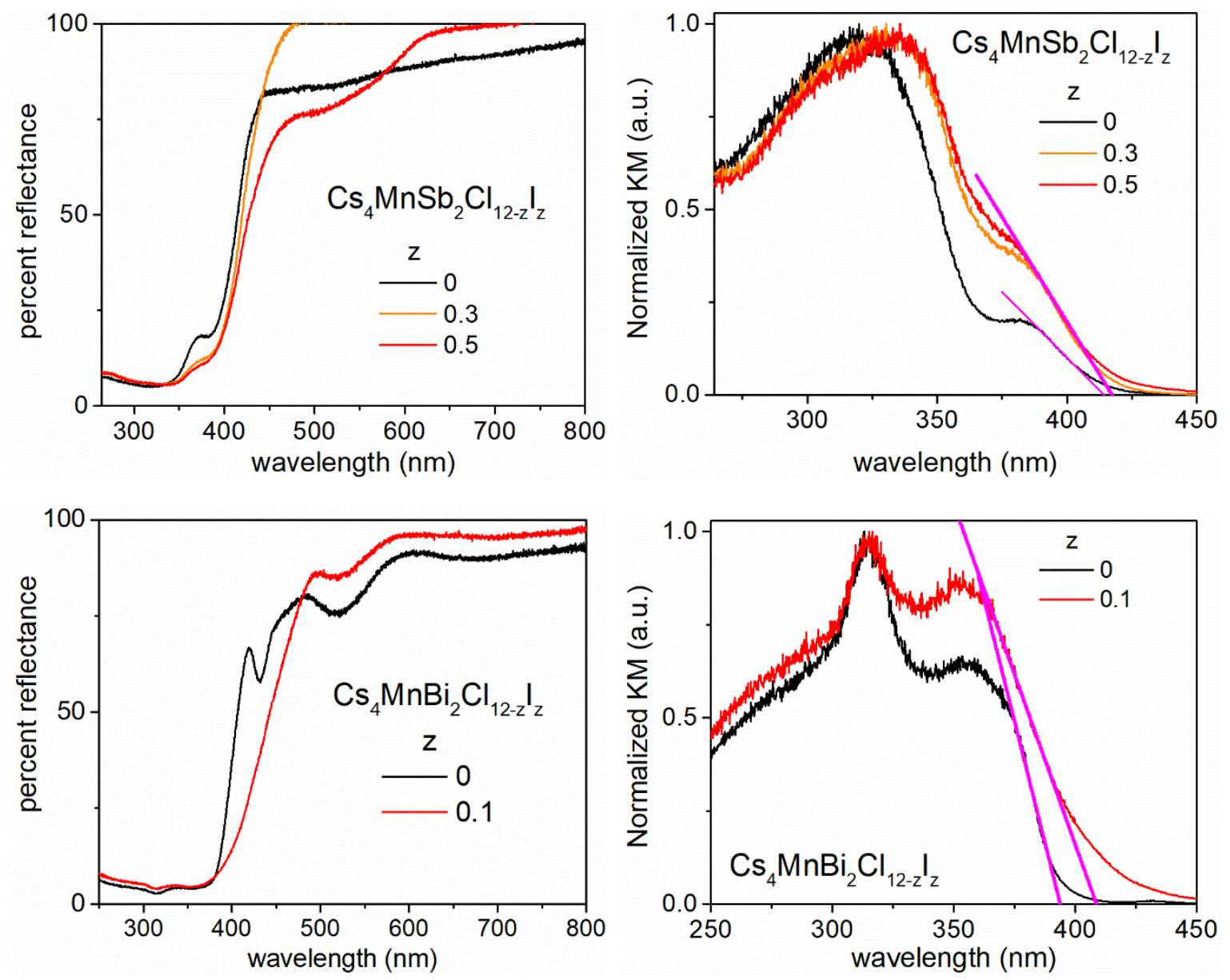

Figure S21. Diffuse reflectance (left column) and pseudo-absorbance (obtained via KubelkaMunk transformation of reflectance data, right column) of halide substituted vacancy ordered quadruple perovskite compositions. Absorption onset fit is shown in magenta. 
Table S5. Summary of anion packing $\left(\mathrm{X}^{-}\right)$and tolerance factors $(t)$ for simple transition metal halide perovskites. $\left(^{*}\right)=$ distorted. $\mathrm{NR}=$ not reported. All radii taken from Shannon ${ }^{5}$ and all structures are from the ICSD. ${ }^{1}$

\begin{tabular}{|c|c|c|c|c|c|c|c|}
\hline \multicolumn{8}{|c|}{ Fluorides } \\
\hline $\mathbf{M}^{2+}$ & Structure & $\mathrm{RbMF}_{3} t$ & ICSD \# & $\mathbf{M}^{2+}$ & Structure & $\mathrm{CsMF}_{3} t$ & ICSD \# \\
\hline $\mathrm{Cd}$ & $3 \mathrm{C}$ & 0.95 & 49587 & $\mathrm{Cd}$ & $3 \mathrm{C}$ & 1.00 & 49582 \\
\hline $\mathrm{Mn}$ & $3 \mathrm{C}$ & 1.00 & 43722 & $\mathrm{Mn}$ & $6 \mathrm{H}$ & 1.05 & 410386 \\
\hline $\mathrm{Fe}$ & $3 C$ & 1.02 & 49586 & $\mathrm{Fe}$ & $6 \mathrm{H}$ & 1.08 & 67169 \\
\hline $\mathrm{Co}$ & $6 \mathrm{H}$ & 1.04 & 410388 & $\mathrm{Co}$ & $9 \mathrm{R}$ & 1.09 & 15091 \\
\hline $\mathrm{Ni}$ & $6 \mathrm{H}$ & 1.07 & 22127 & $\mathrm{Ni}$ & $9 \mathrm{R}$ & 1.12 & 410393 \\
\hline $\mathrm{Cu}$ & $3 C^{*}$ & 1.05 & 69656 & $\mathrm{Cu}$ & NR & 1.10 & $\mathrm{~N} / \mathrm{A}$ \\
\hline $\mathrm{Zn}$ & $6 \mathrm{H}$ & 1.04 & 91835 & $\mathrm{Zn}$ & NR & 1.10 & N/A \\
\hline \multicolumn{8}{|c|}{ Chlorides } \\
\hline $\mathbf{M}^{2+}$ & Structure & $\mathrm{RbMCl}_{3} t$ & ICSD \# & $\mathbf{M}^{2+}$ & Structure & $\mathrm{CsMCl}_{3} \mathrm{t}$ & ICSD \# \\
\hline $\mathrm{Cd}$ & $3 C^{*}$ & 0.90 & 82223 & $\mathrm{Cd}$ & $6 \mathrm{H}$ & 0.95 & 4039 \\
\hline $\mathrm{Mn}$ & $6 \mathrm{H}$ & 0.95 & 54006 & $\mathrm{Mn}$ & $9 \mathrm{R}$ & 0.99 & 2525 \\
\hline $\mathrm{Fe}$ & NR & 0.96 & N/A & $\mathrm{Fe}$ & $2 \mathrm{H}$ & 1.01 & 300249 \\
\hline $\mathrm{Co}$ & $2 \mathrm{H}$ & 0.98 & 24305 & $\mathrm{Co}$ & $2 \mathrm{H}$ & 1.02 & 27511 \\
\hline $\mathrm{Ni}$ & $2 \mathrm{H}$ & 1.00 & 15010 & $\mathrm{Ni}$ & $2 \mathrm{H}$ & 1.04 & 59371 \\
\hline $\mathrm{Cu}$ & $2 \mathrm{H}^{*}$ & 0.98 & 56838 & $\mathrm{Cu}$ & $2 \mathrm{H}^{*}$ & 1.03 & 78435 \\
\hline $\mathrm{Zn}$ & NR & 0.98 & N/A & $\mathrm{Zn}$ & NR & 1.02 & N/A \\
\hline \multicolumn{8}{|c|}{ Bromides } \\
\hline $\mathbf{M}^{2+}$ & Structure & $\operatorname{RbMBr}_{3} t$ & ICSD \# & $\mathbf{M}^{2+}$ & Structure & $\mathrm{CsMBr}_{3} t$ & ICSD \# \\
\hline $\mathrm{Cd}$ & $\mathrm{NH}_{4} \mathrm{CdCl}_{3}$ & 0.89 & 808 & $\mathrm{Cd}$ & $2 \mathrm{H}$ & 0.93 & 281176 \\
\hline $\mathrm{Mn}$ & $2 \mathrm{H}$ & 0.93 & $2 \mathrm{H}$ & $\mathrm{Mn}$ & $2 \mathrm{H}$ & 0.97 & 2782 \\
\hline $\mathrm{Fe}$ & NR & 0.95 & N/A & $\mathrm{Fe}$ & $2 \mathrm{H}$ & 0.99 & 174041 \\
\hline Co & NR & 0.96 & N/A & $\mathrm{Co}$ & NR & 1.00 & N/A \\
\hline $\mathrm{Ni}$ & $2 \mathrm{H}$ & 0.98 & $2 \mathrm{H}$ & $\mathrm{Ni}$ & $2 \mathrm{H}$ & 1.02 & 423829 \\
\hline $\mathrm{Cu}$ & NR & 0.97 & N/A & $\mathrm{Cu}$ & $4 \mathrm{H}$ & 1.01 & 10184 \\
\hline $\mathrm{Zn}$ & NR & 0.96 & N/A & $\mathrm{Zn}$ & NR & 1.01 & N/A \\
\hline \multicolumn{8}{|c|}{ Iodides } \\
\hline $\mathbf{M}^{2+}$ & Structure & $\mathrm{RbMI}_{3} t$ & ICSD \# & $\mathbf{M}^{2+}$ & Structure & $\mathrm{CsMI}_{3} t$ & ICSD \# \\
\hline $\mathrm{Cd}$ & NR & 0.88 & N/A & $\mathrm{Cd}$ & NR & 0.92 & N/A \\
\hline $\mathrm{Mn}$ & NR & 0.91 & N/A & $\mathrm{Mn}$ & $2 \mathrm{H}$ & 0.95 & 15949 \\
\hline $\mathrm{Fe}$ & NR & 0.93 & N/A & $\mathrm{Fe}$ & NR & 0.97 & $\mathrm{~N} / \mathrm{A}$ \\
\hline Co & NR & 0.94 & N/A & Co & NR & 0.98 & N/A \\
\hline $\mathrm{Ni}$ & NR & 0.96 & N/A & $\mathrm{Ni}$ & $2 \mathrm{H}$ & 1.00 & 423830 \\
\hline $\mathrm{Cu}$ & NR & 0.95 & N/A & $\mathrm{Cu}$ & NR & 0.98 & N/A \\
\hline $\mathrm{Zn}$ & NR & 0.94 & N/A & $\mathrm{Zn}$ & NR & 0.98 & N/A \\
\hline
\end{tabular}




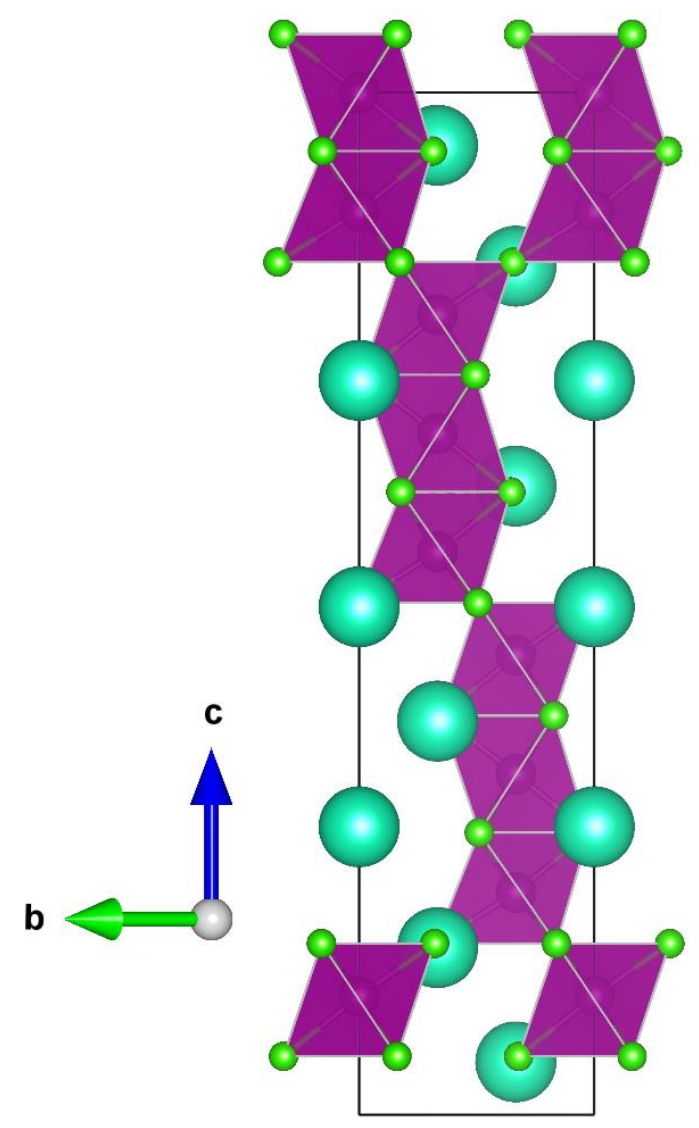

Figure S22. The 9R crystal structure with a 2:1 ratio of hep and ccp anion packing. 


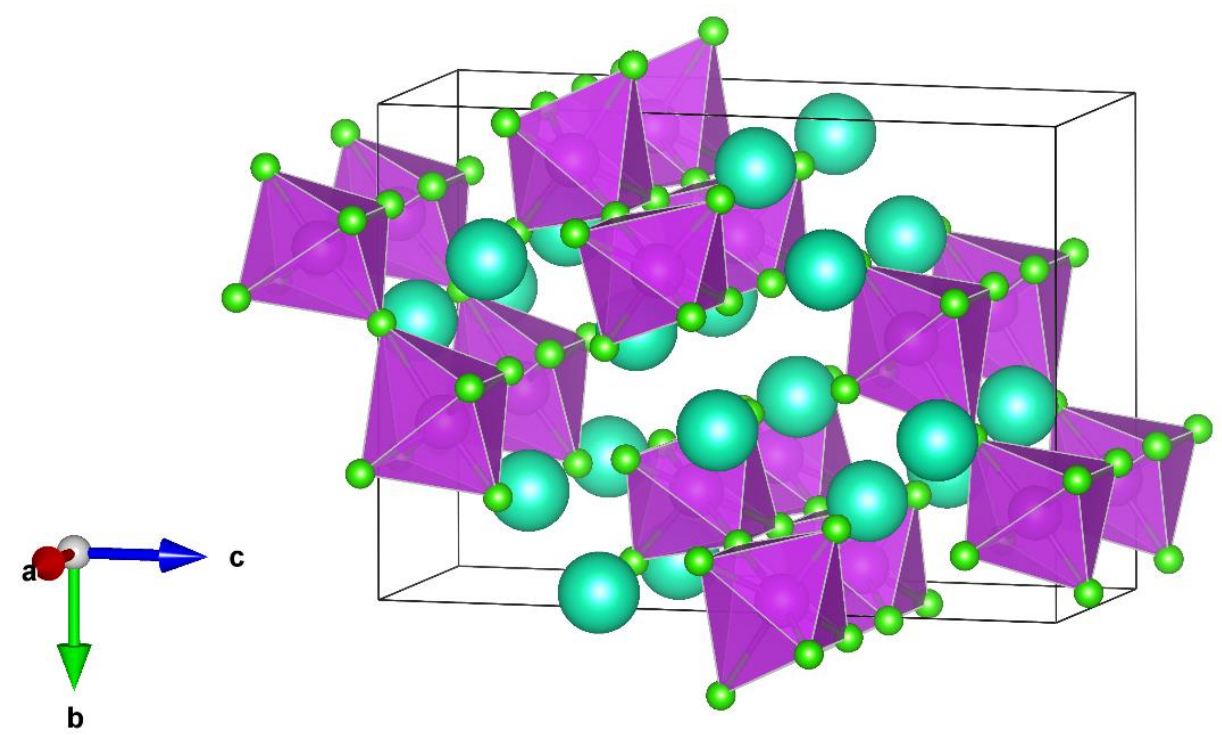

Figure S23. The 1-D chain structure of $\mathrm{Cs}_{3} \mathrm{M}_{2} \mathrm{Cl}_{9}\left(\mathrm{M}^{\mathrm{III}}=\mathrm{Bi}^{3+}, \mathrm{Sb}^{3+}\right)$. 


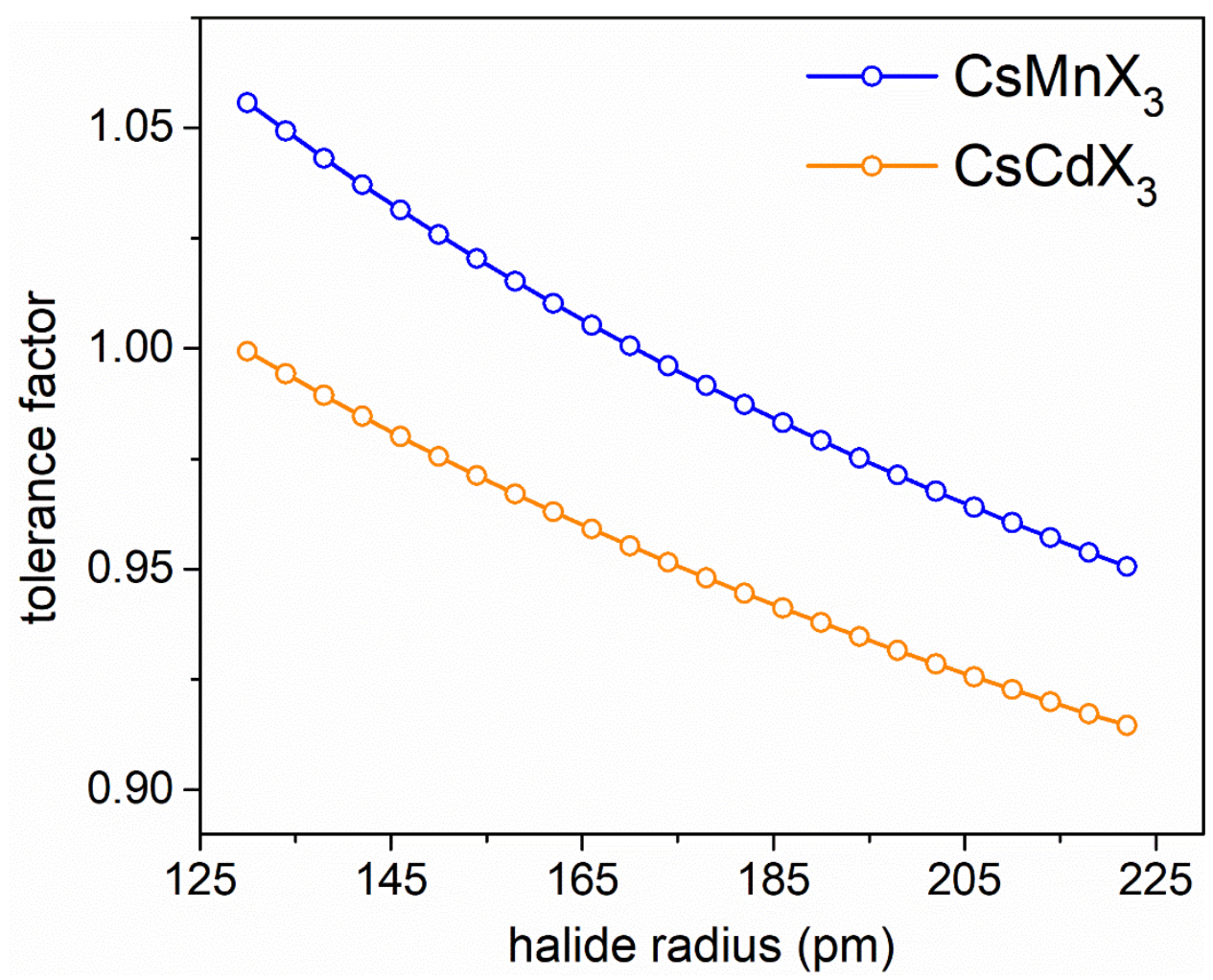

Figure S24. Demonstration of the effect of halide size on the tolerance factor for $\mathrm{CsMnX}_{3}$ and $\mathrm{CsCdX}_{3}$. 


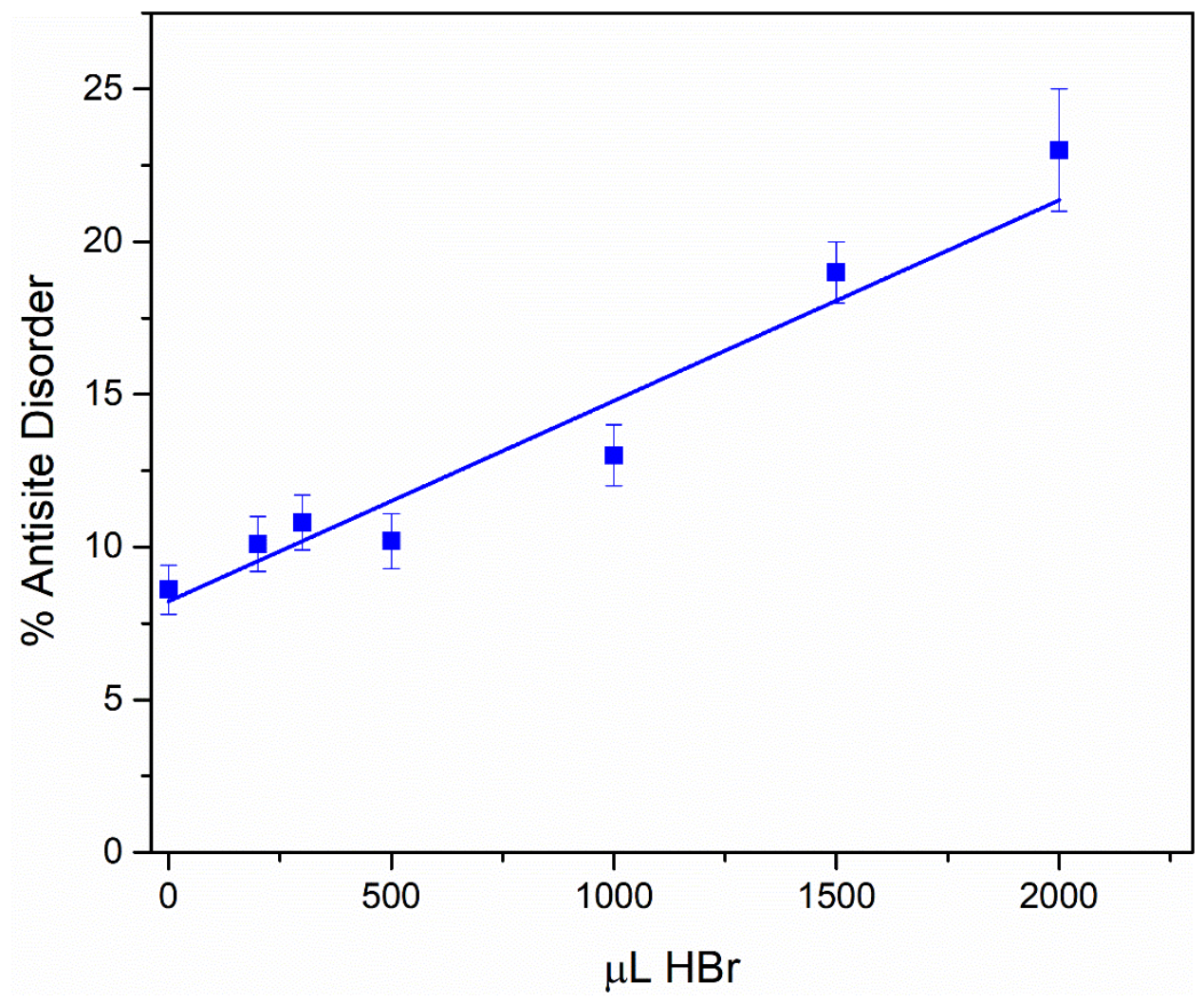

Figure S25. Refined antisite disorder of $\mathrm{Cd}^{2+}$ with respect to volume of $\mathrm{HBr}(\mathrm{aq})$ added to the $\mathrm{Cs}_{4} \mathrm{CdSb}_{2} \mathrm{Cl}_{12-\mathrm{z}} \mathrm{Br}_{\mathrm{z}}$ system. 


\section{References:}

(1) ICSD FIZ Karlsruhe Version 4.4.0 https://icsd.products.fiz-karlsruhe.de/. Accessed 05-152020.

(2) Holzapfel, N. P.; Majher, J. D.; Strom, T. A.; Moore, C. E.; Woodward, P. M. Cs $4 \mathrm{Cd}_{1-}$ ${ }_{x} \mathrm{Mn}_{\mathrm{x}} \mathrm{Bi}_{2} \mathrm{Cl}_{12}$ - $\mathrm{A}$ Vacancy-Ordered Halide Perovskite Phosphor with High-Efficiency Orange-Red Emission. Chem. Mater., 2020, 32, 3510-3516.

(3) Lin, Y.-P.; Hu, S.; Xia, B.; Fan, K.-Q.; Gong, L.-K.; Kong, J.-T.; Huang, X.-Y.; Xiao, Z.; Du, K.-Z. Material Design and Optoelectronic Properties of Three-Dimensional Quadruple Perovskite Halides. J. Phys. Chem. Lett., 2019, 10, 5219-5225.

(4) Vargas, B.; Torres-Cadena, R.; Rodríguez-Hernández, J.; Gembicky, M.; Xie, H.; Jiménez-Mier, J.; Liu, Y.-S.; Menéndez-Proupin, E.; Dunbar, K. R.; Lopez, N.; OlaldeVelasco, P.; Solis-Ibarra, D. Optical, Electronic and Magnetic Engineering of <111> Layered Halide Perovskites. Chem. Mater., 2018, 30, 5315-5321.

(5) Shannon, R. D. Revised Effective Ionic Radii and Systematic Studies of Interatomic Distances in Halides and Chalcogenides, Acta Crystallogr., Sect. A: Cryst. Phys., Diffr., Theor. Gen. Crystallogr., 1976, A32, 751-767. 